\title{
Total synthesis of ent-lepadin F and G by a tandem ene-yne-ene ring closing metathesis
}

\author{
Alexander Niethe, Dirk Fischer and Siegfried Blechert ${ }^{*}$ \\ Institut für Chemie, Technische Universität Berlin, Strasse des 17. Juni 135, D-10623 Berlin, Germany \\ blechert@chem.tu-berlin.de
}

Supporting Information

\section{Table of contents}

General experimental methods

page 1

Experimental procedures for compounds 5, 9, 14-15, 17-22 and 24-25

page $2-9$

Copies of ${ }^{1} \mathrm{H}$ - and ${ }^{13} \mathrm{C}-\mathrm{NMR}$ spectra for all new compounds

page 10 - 47

\section{General experimental methods}

All reagents were obtained commercially and were used without further purification. All reactions were carried out under an inert atmosphere $\left(\mathrm{N}_{2}\right)$ unless otherwise indicated. ${ }^{1} \mathrm{H}$ and ${ }^{13} \mathrm{C}$-NMR spectra were recorded at 500 and $125 \mathrm{MHz}$ in $\mathrm{CDCl}_{3}$ or $\mathrm{C}_{6} \mathrm{D}_{6}$ and chemical shifts are given in ppm relative to the internal solvent peak. Mass spectra were obtained at an ionizing potential of $70 \mathrm{eV}$. IR spectra were measured by attenuated total reflection (ATR) and are reported in wave numbers $\left(\mathrm{cm}^{-1}\right)$. Optical rotations were determined on a polarimeter as solutions in a $10 \mathrm{~cm}$ unit cell at $589 \mathrm{~nm}$. Melting points are uncorrected. 
$26.73 \mathrm{~g}$ (0.30 mol) L-alanin was dissolved in $300 \mathrm{~mL}$ methanol and $32.64 \mathrm{~mL}$ (0.45 mol) thionylchloride was added dropwise at $0{ }^{\circ} \mathrm{C}$. Then the reaction mixture was heated for $1.5 \mathrm{~h}$ under reflux, cooled down an evaporated in vacuo. The resulting white solid was dissolved in $300 \mathrm{~mL}$ methanol, treated with $46 \mathrm{~mL}(9.33 \mathrm{mmol}) \mathrm{Et}_{3} \mathrm{~N}$ at $0{ }^{\circ} \mathrm{C}$ and stirred for $10 \mathrm{~min}$. Afterwards $54.8 \mathrm{~mL}$ (0.45 mol) 4-anisaldehyde and $22.62 \mathrm{~g}(0.36 \mathrm{~mol}) \mathrm{NaCNBH}_{3}$ were added portionwise and stirred additional $16 \mathrm{~h}$ at rt. The solvent was evaporated in vacuo, the residue re-dissolved in $200 \mathrm{~mL}$ methyl tert-butyl ether and $200 \mathrm{~mL}$ of water and extracted with methyl tert-butyl ether (2* $50 \mathrm{~mL}$ ). Reextraction with $1 \mathrm{~N} \mathrm{HCl}$, neutralisation of the acidic aqueous phase with $1 \mathrm{~N} \mathrm{NaOH}$ and again extraction with methyl tert-butyl ether ( $1 * 100 \mathrm{~mL}, 2 * 50 \mathrm{~mL}$ ) performed crude 9 after evaporation in vacuo. Destillation in vacuo $\left(0.8 \mathrm{mbar}, 115^{\circ} \mathrm{C}\right)$ resulted in $41.15 \mathrm{~g}(62 \%) 9$ as colourless liquid.

RF (methyl tert-butyl ether) $=0.38$; ${ }^{1} \mathbf{H}-\mathbf{N M R}\left(500 \mathrm{MHz}, \mathrm{CDCl}_{3}\right): \delta(\mathrm{ppm})=1.30-1.32(\mathrm{~d}, J=7 \mathrm{~Hz}$, 3H), 1.88 (br s, 1H), 3.36-3.40 (q, $J=7 \mathrm{~Hz}, 1 \mathrm{H}$ ), 3.60-3.62 (d, $J=12 \mathrm{~Hz}, 1 \mathrm{H}$ ), 3.72-3.74 (d, $J=12 \mathrm{~Hz}$, 1H), 3.72 (s, 3H), 3.79 (s, 3H), 6.84-6.86 (d, $J=8 \mathrm{~Hz}, 2 \mathrm{H}$ ), 7.23-7.25 (d, $J=8 \mathrm{~Hz}, 2 \mathrm{H})$; ${ }^{13} \mathbf{C}-\mathbf{N M R}$ (125 MHz, $\left.\mathrm{CDCl}_{3}\right): \delta(\mathrm{ppm})=19.1\left(\mathrm{CH}_{3}\right), 51.3\left(\mathrm{CH}_{2}\right), 51.7(\mathrm{CH}), 55.2(\mathrm{CH}), 55.7(\mathrm{CH}), 113.7(\mathrm{CH})$, 129.4 (CH), 131.8 (C), 158.7 (C), 176.2 (C); IR (ATR): v = 3328 (w), 3030 (w), 2993 (w), 2974 (w), 2952 (w), 2936 (w), 2908 (w), 2835 (w), 1733 (s), 1611 (m), 1512 (s), 1442 (m), 1245 (s), 1197 (s), 1174 (s), 1150 (s), 1034 (s); MS (EI, $50^{\circ} \mathrm{C}$ ): m/z (\%) = $222\left(\mathrm{M}^{+},<1\right), 164$ (24), 136 (22), 121 (100), 78 (8); HR-MS $\left(\mathrm{C}_{10} \mathrm{H}_{14} \mathrm{NO}, \mathrm{M}^{+}\right.$-COOMe): calcd. 164.1075, found 164.1073.

\section{S-Hept-1-en-4-ol (17)}

$2.00 \mathrm{~g}$ (21.6 mmol) S-(+)-epichlorohydrin 16 was dissolved in $25 \mathrm{~mL}$ THF cooled to $-78{ }^{\circ} \mathrm{C}$ and treated with $194 \mathrm{mg}$ ( $2.16 \mathrm{mmol}) \mathrm{CuCN}$. Then $32.5 \mathrm{~mL}$ (32.5 mmol) EtMgBr ( $1 \mathrm{M}$ in THF) was added dropwise during a period of $1 \mathrm{~h}$ and the reaction mixture was allowed to warm to $-20{ }^{\circ} \mathrm{C}$ during a period of $4.5 \mathrm{~h}$. $25 \mathrm{~mL}$ saturated aqueous $\mathrm{NH}_{4} \mathrm{Cl}$ was added and extracted with $\mathrm{Et}_{2} \mathrm{O}(3 * 20 \mathrm{~mL})$. The combined organic phases were washed with saturated aqueous $\mathrm{NaCl}$, dried over $\mathrm{Na}_{2} \mathrm{SO}_{4}$ and evaporated in vacuo.

The residue was dissolved in $25 \mathrm{~mL} \mathrm{Et}_{2} \mathrm{O}$, treated with $4.32 \mathrm{~g}$ powdered $\mathrm{NaOH}$ and stirred $16 \mathrm{~h}$ at rt. The suspension was filtered over celite and the filtrate was washed with saturated aqueous $\mathrm{NaCl}$ and dried over $\mathrm{Na}_{2} \mathrm{SO}_{4}$. Then the solvent was evaporated by distillation at normal pressure with a $10 \mathrm{~cm}$ Vigreux-coloumn.

The crude Produkt was again dissolved in $40 \mathrm{~mL}$ THF, cooled to $-78^{\circ} \mathrm{C}$ and treated with $194 \mathrm{mg}$ (2.16 mmol) CuCN. Then $40 \mathrm{~mL}(28.08 \mathrm{mmol})$ vinylMgBr $(0.7 \mathrm{M}$ in THF) was added dropwise during a 
period of $1 \mathrm{~h}$ and the reaction mixture was allowed to warm to $0{ }^{\circ} \mathrm{C}$ during a period of $4 \mathrm{~h}$. $25 \mathrm{~mL}$ saturated aqueous $\mathrm{NH}_{4} \mathrm{Cl}$ was added and extracted with $\mathrm{Et}_{2} \mathrm{O}(3 * 20 \mathrm{~mL})$. The combined organic phases were washed with saturated aqueous $\mathrm{NaCl}$, dried over $\mathrm{Na}_{2} \mathrm{SO}_{4}$ and evaporated in vacuo. Purification by chromatography in hexane/methyl tert-butyl ether $=8: 2$ resulted in $1.41 \mathrm{~g}$ (57\%) of a colourless liquid.

$\mathbf{R F}$ (hexane/EtOAc $=8: 2)=0.23 ;[\alpha]^{\mathbf{2 0}}{ }_{\mathbf{D}}=-10.6^{\circ}\left(\mathrm{c}=1.16, \mathrm{CHCl}_{3}\right) ;{ }^{\mathbf{1}} \mathbf{H}-\mathbf{N M R}\left(500 \mathrm{MHz}, \mathrm{CDCl}_{3}\right)$ : $\delta(\mathrm{ppm})=0.92(\mathrm{t}, J=7.0 \mathrm{~Hz}, 3 \mathrm{H}), 1.32-1.39$ (m, 1H), 1.40-1.50 (m, 3H), 1.65 (br s, 1H), 2.10-2.15 (m, $1 \mathrm{H}), 2.26-2.31$ (m, 1H), 3.62-3.67 (ddt, $J=4.9,7.7,7.4 \mathrm{~Hz}, 1 \mathrm{H}), 5.11-5.13$ (d, $J=11.8 \mathrm{~Hz}, 1 \mathrm{H}), 5.11-$ 5.14 (d, $J=16.2 \mathrm{~Hz}, 1 \mathrm{H}), 5.78-5.86$ (ddt, $J=11.8,16.2,6.7 \mathrm{~Hz}, 1 \mathrm{H}) ;{ }^{13} \mathbf{C}-\mathbf{N M R}\left(125 \mathrm{MHz}, \mathrm{CDCl}_{3}\right)$ : $\delta(\mathrm{ppm})=14.1\left(\mathrm{CH}_{3}\right), 18.9\left(\mathrm{CH}_{2}\right), 39.0\left(\mathrm{CH}_{2}\right), 42.0\left(\mathrm{CH}_{2}\right), 70.4(\mathrm{CH}), 118.1\left(\mathrm{CH}_{2}\right), 135.0(\mathrm{CH}) ; \mathrm{IR}$ (ATR): v = 3369 (br m), 2958 (s), 2930 (s), 2872 (m), 1707 (m), 1642 (w), 1512 (m), 1457 (m), 1365 (m), 1234 (m), 1019 (m).

MS (EI, RT $\left.{ }^{\circ} \mathrm{C}\right): \mathrm{m} / \mathrm{z}(\%)=114\left(\mathrm{M}^{+},<1\right), 73$ (60), 55 (100), 43 (60); HR-MS ( $\left.\mathrm{C}_{7} \mathrm{H}_{14} \mathrm{O}\right)$ : calcd. 114.1044, found 114.1056.

\section{S-4-Methoxymethoxyhept-1-en (18)}

$989 \mathrm{mg}$ (8.66 mmol) 17 was dissolved in $9 \mathrm{~mL}$ dichloromethane, treated at $0{ }^{\circ} \mathrm{C}$ with $\mathrm{mL}$ DIPEA and $1.32 \mathrm{~mL}$ (17.32 mmol) $\mathrm{MOMCl}$ versetzt and stirred $30 \mathrm{~min}$. at $0{ }^{\circ} \mathrm{C}$ and $16 \mathrm{~h}$ at rt. Then $15 \mathrm{~mL} 1 \mathrm{~N}$ $\mathrm{NaOH}$ was added and the mixture was stirred for $10 \mathrm{~min}$., diluted with dichloromethane and washed with $1 \mathrm{~N} \mathrm{HCl}$, water and saturated aqueous $\mathrm{NaHCO}_{3}$, dried over $\mathrm{Na}_{2} \mathrm{SO}_{4}$ and evaporated in vacuo to achieve $1.21 \mathrm{~g}(90 \%)$ of colourless liquid.

$\mathbf{R F}$ (hexane/EtOAc $=8: 2)=0.43 ;[\alpha]^{\mathbf{2 0}}{ }_{\mathbf{D}}=-32 .{ }^{\circ}\left(\mathrm{c}=1.18, \mathrm{CHCl}_{3}\right) ;{ }^{1} \mathbf{H}-\mathbf{N M R}\left(500 \mathrm{MHz}, \mathrm{CDCl}_{3}\right)$ : $\delta(\mathrm{ppm})=0.90(\mathrm{t}, J=7.2 \mathrm{~Hz}, 3 \mathrm{H}), 1.27-1.50(\mathrm{~m}, 4 \mathrm{H}), 2.25-2.28(\mathrm{dd}, J=5.9,6.9 \mathrm{~Hz}, 2 \mathrm{H}), 3.36(\mathrm{~s}, 3 \mathrm{H})$, 3.57-3.62 (m, 1H), 4.61-4.63 (d, $J=7.0 \mathrm{~Hz}, 1 \mathrm{H}), 4.66-4.67$ (d, $J=7.0 \mathrm{~Hz}, 1 \mathrm{H}), 5.02-5.04$ (d, $J=9.1$ Hz, 1H), 5.04-5.08 (d, $J=17.2 \mathrm{~Hz}, 1 \mathrm{H}), 5.76-5.85$ (ddt, $J=10.1,17.2,7.1 \mathrm{~Hz}, 1 \mathrm{H}) ;{ }^{13}$ C-NMR (125 $\left.\mathrm{MHz}, \mathrm{CDCl}_{3}\right): \delta(\mathrm{ppm})=14.1\left(\mathrm{CH}_{3}\right), 18.6\left(\mathrm{CH}_{2}\right), 36.4\left(\mathrm{CH}_{2}\right), 38.9\left(\mathrm{CH}_{2}\right), 55.5\left(\mathrm{CH}_{3}\right), 76.6(\mathrm{CH}), 95.4$ ( $\left.\mathrm{CH}_{2}\right), 117.0\left(\mathrm{CH}_{2}\right), 134.9(\mathrm{CH})$; IR (ATR): v = 2960 (m), 2928 (m), 2872 (m), $1715(\mathrm{w}), 1642$ (w), 1458 (w), 1365 (w), 1260 (m), 1096 (s), 1040 (s); MS (EI, RT $\left.{ }^{\circ} \mathrm{C}\right): \mathrm{m} / \mathrm{z}(\%)=127\left(\mathrm{M}^{+}-\mathrm{OCH}_{3}, 4\right), 117$ (96), 97 (16), 71 (40), 55 (100); HR-MS $\left(\mathrm{C}_{8} \mathrm{H}_{15} \mathrm{O}, \mathrm{M}^{+}-\mathrm{OCH}_{3}\right)$ : calcd. 127.1122, found 127.1131. 
$1.21 \mathrm{~g}$ (7.65 mmol) 18 was dissolved in $40 \mathrm{~mL} \mathrm{THF}$, cooled to $\quad-20{ }^{\circ} \mathrm{C}$ and then $76.5 \mathrm{~mL}$ (76.5 mmol) $\mathrm{BH}_{3} \mathrm{xTHF}$ ( $1 \mathrm{M}$ in THF) was added dropwise. The reaction was allowed to warm up to $\mathrm{rt}$ overnight, was recooled to $0^{\circ} \mathrm{C}$ and treated with $20 \mathrm{~mL} \mathrm{H}_{2} \mathrm{O}_{2}(30 \%)$ and $40 \mathrm{~mL} 3 \mathrm{~N} \mathrm{NaOH}$ versetzt. After stirring for additional $1 \mathrm{~h}$ at rt excess borane was carefully neutralised by addition of $25 \mathrm{~g} \mathrm{Na}_{2} \mathrm{SO}_{3}$ dissolved in $25 \mathrm{~mL}$ water. The phases were separated, extracted with methyl tert-butyl ether and washed wit water and saturated aqueous $\mathrm{NaCl}$, dried over $\mathrm{Na}_{2} \mathrm{SO}_{4}$ and evaporated in vacuo. Purification by chromatography in hexane/methyl tert-butyl ether = 7:3 and 6:4 gave $787 \mathrm{mg}$ (58\%) 19 as colourless liquid.

$\mathbf{R F}$ (hexane/EtOAc $=8: 2)=0.37 ;[\alpha]^{\mathbf{2 0}}{ }_{\mathbf{D}}=6.3^{\circ}\left(\mathrm{c}=1.97, \mathrm{CHCl}_{3}\right) ;{ }^{1} \mathbf{H}-\mathbf{N M R}\left(500 \mathrm{MHz}, \mathrm{CDCl}_{3}\right)$ : $\delta(\mathrm{ppm})=0.89(\mathrm{t}, J=7.1 \mathrm{~Hz}, 3 \mathrm{H}), 1.27-1.39$ (m, 2H), 1.40-1.66 (m, 6H), 2.06 (br s, 1H), 3.36 (s, 3H), 3.55-3.59 (m, 1H), 3.40-3.66 (m, 2H), 4.64 (s, 2H); ${ }^{13}$ C-NMR (125 MHz, $\left.\mathrm{CDCl}_{3}\right): \delta(\mathrm{ppm})=14.2$ $\left(\mathrm{CH}_{3}\right)$, $18.6\left(\mathrm{CH}_{2}\right)$, 28.3, 30.6, $36.4\left(\mathrm{CH}_{2}\right), 55.6\left(\mathrm{CH}_{3}\right), 63.0\left(\mathrm{CH}_{2}\right), 77.1(\mathrm{CH}), 95.4\left(\mathrm{CH}_{2}\right)$; IR $(\mathrm{ATR}): v$ = 4321 (br m), 2956 (m), 2934 (m), 2874 (m), 1454 (w), 1379 (w), 1152 (m), 1096 (m), 1039 (s); MS (EI, RT $\left.{ }^{\circ} \mathrm{C}\right): \mathrm{m} / \mathrm{z}(\%)=133\left(\mathrm{M}^{+}-\mathrm{C}_{3} \mathrm{H}_{7},<1\right), 117$ (24), 101 (14), 97 (10), 71 (100), 55 (36); HR-MS $\left(\mathrm{C}_{6} \mathrm{H}_{13} \mathrm{O}_{3}, \mathrm{M}^{+}-\mathrm{C}_{3} \mathrm{H}_{7}\right)$ : calcd. 133.0864, found 133.0855 .

\section{S-5-(4-Methoxymethoxyheptylsulfanyl)-1-phenyl-1H-tetrazole (20)}

$568 \mathrm{mg}$ (3.22 mmol) 19, $632 \mathrm{mg}$ (3.54 mmol) 1-phenyl-1H-tetrazole-5-thiol and $1.10 \mathrm{~g}$ (4.19 mmol) triphenylphosphine were dissolved in $30 \mathrm{~mL}$ THF. $0.76 \mathrm{~mL}$ (3.86 mmol) DIAD in $10 \mathrm{~mL}$ THF was added dropwise at $0{ }^{\circ} \mathrm{C}$ and the mixtures was allowed to warm up to rt overnight. The resulting suspension was filtered over celite and the filtrate was evaporated in vacuo. Purification by chromatography in hexane/EtOAc = 9:1 gave 969 mg (89\%) 20 as light yellow oil.

$\mathbf{R F}$ (hexane/EtOAc $=8: 2)=0.22 ;[\alpha]^{\mathbf{2 0}}{ }_{\mathbf{D}}=3.4^{\circ}\left(\mathrm{c}=1.27, \mathrm{CHCl}_{3}\right) ;{ }^{\mathbf{1}} \mathbf{H}-\mathbf{N M R}\left(500 \mathrm{MHz}, \mathrm{CDCl}_{3}\right)$ : $\delta(\mathrm{ppm})=0.90(\mathrm{t}, J=7.1 \mathrm{~Hz}, 3 \mathrm{H}), 1.25-1.38$ (m, 2H), 1.38-1.54 (m, 2H), 1.58-1.69 (m, 2H), 1.83-1.98 (m, 2H), 3.35 (s, 3H), 3.37-3.45 (m, 2H), 3.53-3.58 (m, 1H), 4.60-4.64 (m, 2H), 7.51-7.58 (m, 5H); ${ }^{13} \mathrm{C}-$ NMR (125 MHz, $\left.\mathrm{CDCl}_{3}\right): \delta(\mathrm{ppm})=14.2\left(\mathrm{CH}_{3}\right), 18.5\left(\mathrm{CH}_{2}\right), 25.0\left(\mathrm{CH}_{2}\right), 33.2\left(\mathrm{CH}_{2}\right), 33.5\left(\mathrm{CH}_{2}\right), 36.4$ ( $\left.\mathrm{CH}_{2}\right)$, $55.6\left(\mathrm{CH}_{3}\right), 76.7(\mathrm{CH}), 95.5\left(\mathrm{CH}_{2}\right), 123.9$ (2x CH), 129.8 (2x CH), $130.1(\mathrm{CH}), 133.7(\mathrm{C}), 154.4$ (C); IR (ATR): v = 2955 (m), 2932 (m), 2873 (m), 1597 (w), 1500 (s), 1387 (w), 1146 (m), 1095 (m), 1039 (s); MS (EI, 140 C): m/z (\%) = 336 (M+, 4), 293 (90), 275 (44), 217 (40), 193 (40), 161 (70), 118 (100), 87 (76), 77 (90); HR-MS $\left(\mathrm{C}_{16} \mathrm{H}_{24} \mathrm{~N}_{4} \mathrm{SO}_{2}\right)$ : calcd. 336.1619, found 336.1622. 
$932 \mathrm{mg}$ (2.77 mmol) 20 was dissolved in $60 \mathrm{~mL}$ dichloromethane, treated with $3.19 \mathrm{~g}$ (13.85 mmol) mCPBA and stirred at rt for $3 \mathrm{~d}$. Then the mixture was carefully quenched by addition of aqueous $\mathrm{Na}_{2} \mathrm{SO}_{3}$ (15 mmol) and stirring for $10 \mathrm{~min}$. The phases were separated and extracted with dichloromethane, washed with $1 \mathrm{~N} \mathrm{NaOH}$, water and saturated aqueous $\mathrm{NaCl}$, dried $\mathrm{Na}_{2} \mathrm{SO}_{4}$ and evaporated in vacuo to achieve $970 \mathrm{mg}$ (95\%) yellow oil.

$\mathbf{R F}$ (hexane/EtOAc $=8: 2)=0.30 ;[\alpha]^{\mathbf{2 0}}{ }_{\mathbf{D}}=9.5^{\circ}\left(\mathrm{c}=1.19, \mathrm{CHCl}_{3}\right) ;{ }^{\mathbf{1}} \mathbf{H}-\mathbf{N M R}\left(500 \mathrm{MHz}, \mathrm{CDCl}_{3}\right)$ : $\delta(\mathrm{ppm})=0.91(\mathrm{t}, J=7.1 \mathrm{~Hz}, 3 \mathrm{H}), 1.29-1.38$ (m, 2H), 1.39-1.47 (m, 1H), 1.50-1.57 (m, 1H), 1.62-1.76 (m, 2H), 1.98-2.12 (m, 2H), 3.36 (s, 3H), 3.55-3.60 (m, 1H), 3.72-3.83 (m, 2H), 4.60-4.65 (m, 2H), 7.57-7.64 (m, 3H), 7.68-7.70 (m, 2H); ${ }^{13} \mathrm{C}-\mathbf{N M R}\left(125 \mathrm{MHz}, \mathrm{CDCl}_{3}\right): \delta(\mathrm{ppm})=14.2\left(\mathrm{CH}_{3}\right), 18.1\left(\mathrm{CH}_{2}\right)$, $18.5\left(\mathrm{CH}_{2}\right), 32.6\left(\mathrm{CH}_{2}\right), 36.4\left(\mathrm{CH}_{2}\right), 55.8\left(\mathrm{CH}_{3}\right), 56.1\left(\mathrm{CH}_{2}\right), 76.5(\mathrm{CH}), 95.6\left(\mathrm{CH}_{2}\right), 125.1(2 \mathrm{x} \mathrm{CH})$, 129.7 (2x CH), 131.5 (CH), 133.1 (C), 153.5 (C); IR (ATR): v = 2957 (m), 2934 (m), 2874 (w), 1769 (w), 1720 (w), 1595 (w), 1498 (m), 1340 (s), 1151 (s), 1096 (m), 1035 (s); MS (EI, $\left.130{ }^{\circ} \mathrm{C}\right): \mathrm{m} / \mathrm{z}(\%)=$ 367 ( $\left.\mathrm{M}^{+},<1\right), 325$ (100), 307 (12), 204 (12), 132 (30), 118 (100), 97 (20), 77 (36), 65 (40), 55 (48); HR-MS $\left(\mathrm{C}_{16} \mathrm{H}_{23} \mathrm{~N}_{4} \mathrm{SO}_{4}\right)$ : calcd. 367.1440, found 367.1449.

\section{S,4S-2-[[1-(3-Benzyloxy-prop-1-ynyl)-hex-4-enyl]-(4-methoxy-benzyl)-amino]-propionic}

\section{aldehyde (14)}

$4.75 \mathrm{~mL}$ (56.17 mmol) oxallylchloride was dissolved in $500 \mathrm{~mL}$ dichloromethane and treated at $-78{ }^{\circ} \mathrm{C}$ with $7.98 \mathrm{~mL}$ (112.34 mmol) DMSO in $20 \mathrm{~mL}$ dichloromethane. The solution was stirred for $30 \mathrm{~min}$. at ambient temperature and then $19.73 \mathrm{~g}$ (46.81 mmol) 12 dissolved in $50 \mathrm{~mL}$ dichloromethane was added dropwise and stirred $2.5 \mathrm{~h}$ at $-78{ }^{\circ} \mathrm{C}$. Then $30.01 \mathrm{~mL}(215.33 \mathrm{mmol}) \mathrm{Et}_{3} \mathrm{~N}$ was added and the reaction mixture was allowed to warm up to rt overnight. Afterwards the solution was washed with $100 \mathrm{~mL}$ water, $100 \mathrm{~mL} 2.5 \mathrm{~N} \mathrm{HCl}$ and $100 \mathrm{~mL}$ saturated aqueous $\mathrm{NaCl}$, dried over $\mathrm{Na}_{2} \mathrm{SO}_{4}$ and evaporated in vacuo to give $19.52 \mathrm{~g}$ (99\%) of a yellow oil which was used without further purification.

RF (hexane/methyl tert-butyl ether $=1: 1)=0.56 ;{ }^{1} \mathbf{H}-\mathbf{N M R}\left(500 \mathrm{MHz}, \mathrm{CDCl}_{3}\right): \delta(\mathrm{ppm})=1.24-1.26(\mathrm{~d}$, $J=7.0 \mathrm{~Hz}, 3 \mathrm{H}), 1.59-1.61(\mathrm{~m}, 3 \mathrm{H}), 1.67-1.79(\mathrm{~m}, 2 \mathrm{H}), 2.16-2.21(\mathrm{dt}, J=7.4,7.6 \mathrm{~Hz}, 2 \mathrm{H}), 3.39-3.45$ (q, $J=7.2 \mathrm{~Hz}, 1 \mathrm{H}), 3.60-3.64$ (m, 1H), 3.78-3.82 (d, $J=14.1 \mathrm{~Hz}, 1 \mathrm{H}), 3.79$ (s, 3H), 3.84-3.87 (d, $J=14.1$ 
Hz, 1H), 4.22 (s, 2H), 4.60 (s, 2H), 5.29-5.36 (m, 1H), 5.42-5.50 (m, 1H), 6.82-6.86 (m, 2H), 7.24-7.27

(m, 2H), 7.28-7.34 (m, 1H), 7.35-7.38 (m, 4H), 9.84 (d, $J=1 \mathrm{~Hz}, 1 \mathrm{H})$.

\section{R,4S,6S-4-[[1-(3-Benzyloxy-prop-1-ynyl)-hex-4-enyl]-(4-methoxy-benzyl)-amino]-pent-1-en-3-ol}

\section{(5)}

$19.52 \mathrm{~g}$ (46.64 mmol) 14 was dissolved in $500 \mathrm{~mL}$ THF, and $41.34 \mathrm{~mL}$ (70.00 mmol) vinylmagnesiumchloride was added dropwise at $-78^{\circ} \mathrm{C}$. The mixture was allowed to warm up to rt overnight and then water was added and extracted with methyl tert-butyl ether $(3 * 50 \mathrm{~mL})$. The organic phase was washed with saturated aqueous $\mathrm{NaCl}$, dried over $\mathrm{Na}_{2} \mathrm{SO}_{4}$ and evaporated in vacuo. Purification by chromatography in hexane/methyl tert-butyl ether = 7:3 gave $13.07 \mathrm{~g}(63 \%) 5$ as colourless oil.

$\mathbf{R F}$ (hexane/methyl tert-butyl ether $=1: 1)=0.46 ;[\boldsymbol{\alpha}]^{\mathbf{2 0}}{ }_{\mathbf{D}}=-30.7^{\circ}\left(\mathrm{c}=0.94, \mathrm{CHCl}_{3}\right) ;{ }^{\mathbf{1}} \mathbf{H}-\mathbf{N M R}(500$ $\left.\mathrm{MHz}, \mathrm{CDCl}_{3}\right): \delta(\mathrm{ppm})=1.08-1.10(\mathrm{~d}, J=7.0 \mathrm{~Hz}, 3 \mathrm{H}), 1.59-1.62(\mathrm{~m}, 4 \mathrm{H}), 1.66-1.75(\mathrm{~m}, 1 \mathrm{H}), 2.13-$ 2.18 (dt, $J=7.4,7.1 \mathrm{~Hz}, 2 \mathrm{H}), 2.76$ (br s, $1 \mathrm{H}), 3.00-3.06(\mathrm{dq}, J=3.3,6.9 \mathrm{~Hz}, 1 \mathrm{H}), 3.75-3.84(\mathrm{~m}, 4 \mathrm{H})$, 4.26 (s, 2H), 4.44-4.48 (m, 1H), 4.63 (s, 2H), 5.08-5.12 (dt, $J=10.6,1.7 \mathrm{~Hz}, 1 \mathrm{H}), 5.25-5.31(\mathrm{dt}, J=$ 17.2, $1.8 \mathrm{~Hz}, 1 \mathrm{H}), 5.31-5.37$ (m, 1H), 5.42-5.50 (m, 1H), 5.79-5.88 (ddd, $J=17.2,10.6,4.9 \mathrm{~Hz}, 1 \mathrm{H}$ ), 6.84-6.86 (m, 2H), 7.22-7.26 (m, 2H), 7.28-7.34 (m, 1H), 7.35-7.46 (m, 4H); ${ }^{13}$ C-NMR (125 MHz, $\left.\mathrm{CDCl}_{3}\right): \delta(\mathrm{ppm})=12.5\left(\mathrm{CH}_{3}\right), 12.8\left(\mathrm{CH}_{3}\right), 24.0\left(\mathrm{CH}_{2}\right), 33.8\left(\mathrm{CH}_{2}\right), 50.3\left(\mathrm{CH}_{2}\right), 51.1(\mathrm{CH}), 55.2(\mathrm{CH})$, $57.5\left(\mathrm{CH}_{2}\right), 58.4(\mathrm{CH}), 71.5\left(\mathrm{CH}_{2}\right), 71.6(\mathrm{CH}), 80.5(\mathrm{C}), 87.1(\mathrm{C}), 113.7(\mathrm{CH}), 114.6\left(\mathrm{CH}_{2}\right), 124.8(\mathrm{CH})$, 127.8 (CH), 128.0 (2x CH), 128.4 (2x CH), 129.3 (CH), $129.4(\mathrm{CH}), 130.1$ (2x CH), 132.3 (C), 137.5 (C), 139.0 (CH), 158.6 (C); IR (ATR): v = 3549 (br m), 3463 (br m), 3010 (m), 2934 (m), 2856 (m), 1611 (m), 1511 (s), 1244 (s), 1072 (s); MS (EI, $\left.200{ }^{\circ} \mathrm{C}\right): \mathrm{m} / \mathrm{z}(\%)=446$ (M+ 4 ), 390 (100), 121 (100), 91 (70); HR-MS $\left(\mathrm{C}_{29} \mathrm{H}_{36} \mathrm{NO}_{3}, \mathrm{M}^{+}-\mathrm{H}\right)$ : calcd. 446.2695, found 446.3694; Anal. calcd. for $\mathrm{C}_{29} \mathrm{H}_{37} \mathrm{NO}_{3}$ : C 77.82\%, H 8.33\%, N 3.13\%; found C 77.58\%, H 8.33\%, N 3.13\%. 


\section{1,2,3,7,8,8a-hexahydro-quinoline (15)}

$1.01 \mathrm{~g}$ (2.50 mmol) 4 was dissolved in $25 \mathrm{~mL}$ dichlormethane, treated with $338 \mathrm{mg}$ (5.00 mmol) imidazole and $563 \mathrm{mg}$ (3.75 mmol) TBDMSCl and stirred for $18 \mathrm{~h}$ at rt. The remaining white suspension was filtered over celite, the filtrate was washed with $1 \mathrm{~N} \mathrm{HCl}$, water and saturated aqueous $\mathrm{NaHCO}_{3}$, dried over $\mathrm{Na}_{2} \mathrm{SO}_{4}$ and evaporated in vacuo. The resulting brown oil (1.34 g, 100\%) was used without further purification.

RF (hexane/EtOAc $=6: 4)=0.66 ;{ }^{1} \mathbf{H}-\mathbf{N M R}\left(500 \mathrm{MHz}, \mathrm{CDCl}_{3}\right): \delta(\mathrm{ppm})=0.01(\mathrm{~s}, 3 \mathrm{H}), 0.06(\mathrm{~s}, 3 \mathrm{H})$, 0.87 (s, 9H), 0.93-0.95 (d, $J=6.7 \mathrm{~Hz}, 3 \mathrm{H}), 1.52-1.57$ (m, 1H), 1.98-2.04 (m, 1H), 2.18-2.24 (m, 2H), 2.88-2.94 (dq, $J=2.8,6.7 \mathrm{~Hz}, 1 \mathrm{H}), 3.08-3.11$ (br d, $J=12.0 \mathrm{~Hz}, 1 \mathrm{H}), 3.55-3.58(\mathrm{~d}, J=14.5 \mathrm{~Hz}, 1 \mathrm{H})$, 3.79 (s, 3H), 3.87-3.91 (d, $J=14.5 \mathrm{~Hz}, 1 \mathrm{H}), 3.89-3.92(\mathrm{~m}, 1 \mathrm{H}), 4.08-4.11$ (d, $J=11.6 \mathrm{~Hz}, 1 \mathrm{H}), 4.17-$ 4.20 (d, $J=11.6 \mathrm{~Hz}, 1 \mathrm{H}), 4.49$ (s, 2H), 5.74-5.75 (br d, $J=4.5 \mathrm{~Hz}, 1 \mathrm{H}), 5.85-5.88$ (m, 1H), 6.81-6.83 (m, 2H), 7.27-7.31 (m, 1H), 7.33-7.38 (m, 6H).

\section{S,3R,4aR,5R,8aS-3-(tert-Butyl-dimethyl-silanyloxy)-5-formyl-2-methyloctahydroquinoline-1- carboxylic acid-tert-butyl ester (22)}

$0.06 \mathrm{~mL}$ (0.72 mmol) Oxalylchloride was dissolved in $4 \mathrm{~mL}$ dichloromethane and treated at $-78{ }^{\circ} \mathrm{C}$ with $0.10 \mathrm{~mL}$ (1.44 mmol) DMSO in $1 \mathrm{~mL}$ dichloromethane. The solution was stirred for $30 \mathrm{~min}$. at ambient temperature and then $150 \mathrm{mg}(0.36 \mathrm{mmol}) 3$ dissolved in $1 \mathrm{~mL}$ dichloromethane was added dropwise and stirred $3 \mathrm{~h}$ at $-78{ }^{\circ} \mathrm{C}$. Then $0.3 \mathrm{~mL}(2.16 \mathrm{mmol}) \mathrm{Et}_{3} \mathrm{~N}$ was added and the reaction mixture was allowed to warm up to rt overnight. Afterwards the solution was washed with water, $2.5 \mathrm{~N} \mathrm{HCl}$ and saturated aqueous $\mathrm{NaHCO}_{3}$, dried over $\mathrm{Na}_{2} \mathrm{SO}_{4}$ and evaporated in vacuo to give $137 \mathrm{mg}(92 \%)$ white crystals which were used without further purification.

$\mathbf{R F}($ hexane/EtOAc $=8: 2)=0.53 ;[\boldsymbol{\alpha}]^{\mathbf{2 0}}{ }_{\mathbf{D}}=-18.2^{\circ}\left(\mathrm{c}=0.83, \mathrm{CHCl}_{3}\right) ; \mathbf{M P} 75{ }^{\circ} \mathrm{C} ;{ }^{\mathbf{1}} \mathbf{H}-\mathbf{N M R}(500 \mathrm{MHz}$, $\left.\mathrm{CDCl}_{3}\right): \delta(\mathrm{ppm})=0.03(\mathrm{~s}, 6 \mathrm{H}), 0.86(\mathrm{~s}, 9 \mathrm{H}), 1.21-1.22(\mathrm{~d}, J=6.9 \mathrm{~Hz}, 3 \mathrm{H}), 1.24-1.33(\mathrm{~m}, 1 \mathrm{H}), 1.43-$ 
1.50 (m, 2H), 1.45 (s, 9H), 1.74-1.83 (m, 3H), 1.85-1.91 (ddd. $J=7.3,7.3,14.6$ Hz, 1H), 1.99-2.08 (m, 1H), 2.42-2.47 (dt, $J=3.7,12.5 \mathrm{~Hz}, 1 \mathrm{H}), 2.70-2.77$ (dddd, $J=4.5,45,4.5,4.4,8.0 \mathrm{~Hz}, 1 \mathrm{H}), 3.74-3.79$ (m, 1H), 3.79-3.83 (m, 1H), 3.83-3.88 (m, 1H); ${ }^{13} \mathbf{C}-\mathbf{N M R}\left(125 \mathrm{MHz}, \mathrm{CDCl}_{3}\right): \delta(\mathrm{ppm})=-4.9\left(\mathrm{CH}_{3}\right),-$ $4.7\left(\mathrm{CH}_{3}\right), 17.9(\mathrm{C}), 19.5\left(\mathrm{CH}_{3}\right), 19.7\left(\mathrm{CH}_{2}\right), 23.7\left(\mathrm{CH}_{2}\right), 25.6\left(\mathrm{CH}_{2}\right), 25.7\left(3 \mathrm{x} \mathrm{CH}_{3}\right), 27.2\left(\mathrm{CH}_{2}\right), 28.5$ (3x $\left.\mathrm{CH}_{3}\right), 30.8(\mathrm{CH})$, 53.3, $53.4(\mathrm{CH}), 54.3(\mathrm{CH}), 70.2(\mathrm{CH}), 79.3(\mathrm{C}), 155.5(\mathrm{C}), 203.6(\mathrm{CH}) ; \mathrm{IR}$ (ATR): v = 2954 (s), 2930 (s), 2857 (m), 1725 (m), 1686 (s), 1395 (s), 1365 (s), 1254 (m), 1172 (m),

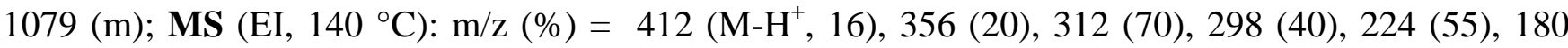
(40); HR-MS $\left(\mathrm{C}_{22} \mathrm{H}_{42} \mathrm{NO}_{4} \mathrm{Si}, \mathrm{M}-\mathrm{H}^{+}\right)$: calcd. 412.2883, found 412.2882.

\section{S,3R,4aR,5R,8aS,5’S-3-(tert-Butyl-dimethyl-silanyloxy)-5-(5-methoxymethoxyoct-1-enyl)-2- methyloctahydroquinoline-1-carboxylic acid tert-butyl ester (24)}

$115 \mathrm{mg}(0.21 \mathrm{mmol}) 23$ was dissolved in $5 \mathrm{~mL}$ methanol, $11 \mathrm{mg}(0.01 \mathrm{mmol}) \mathrm{Pd} / \mathrm{C}(10 \%)$ was added and the mixture was stirred for $18 \mathrm{~h}$ under a hydrogen atmosphere. The catalyst was separated by filtration over celite and the filtrate was evaporated in vacuo. The residue was dissolved in $10 \mathrm{~mL}$ THF treated with $332 \mathrm{mg}(1.05 \mathrm{mmol}) \mathrm{TBAF}^{*} 2 \mathrm{H}_{2} \mathrm{O}$ and stirred $16 \mathrm{~h}$ at $60{ }^{\circ} \mathrm{C}$. Afterwards the mixture was diluted with methyl tert-butyl ether and washed with water and saturated aqueous $\mathrm{NaCl}$, dried over $\mathrm{Na}_{2} \mathrm{SO}_{4}$ and evaporated in vacuo. Purification by chromatography in hexane/EtOAc = 9:1 gave $73 \mathrm{mg}$ (79\%) 24 as colourless oil.

$\mathbf{R F}$ (hexane/EtOAc $=6: 4)=0.44 ;[\alpha]^{\mathbf{2 0}}{ }_{\mathbf{D}}=+5.2^{\circ}\left(\mathrm{c}=0.30, \mathrm{CHCl}_{3}\right) ;{ }^{\mathbf{1}} \mathbf{H}-\mathbf{N M R}\left(500 \mathrm{MHz}, \mathrm{CDCl}_{3}\right)$ : $\delta(\mathrm{ppm})=0.90-0.93(\mathrm{t}, J=7.1 \mathrm{~Hz}, 3 \mathrm{H}), 1.05-1.14(\mathrm{~m}, 1 \mathrm{H}), 1.15-1.21(\mathrm{~m}, 2 \mathrm{H}), 1.23-1.31(\mathrm{~m}, 2 \mathrm{H}), 1.27-$ $1.28(\mathrm{~d}, J=6.8 \mathrm{~Hz}, 3 \mathrm{H}), 1.31-1.39(\mathrm{~m}, 3 \mathrm{H}), 1.39-1.44(\mathrm{~m}, 3 \mathrm{H}), 1.44-1.52(\mathrm{~m}, 14 \mathrm{H}), 1.61$ (br s, $1 \mathrm{H})$, 1.68-1.77 (m, 2H), 1.77-1.88 (m, 2H), 2.11-2.18 (ddd, $J=4.6,11.1,11.3 \mathrm{~Hz}, 1 \mathrm{H}), 3.38$ (s, 3H), 3.503.54 (dddd, $J=5.8,5.8,5.9,5.9 \mathrm{~Hz}, 1 \mathrm{H}), 3.74-3.82$ (m, 2H), 3.85-3.89 (ddd, $J=3.8,3.8,7.4 \mathrm{~Hz}, 1 \mathrm{H})$, $4.65(\mathrm{~s}, 2 \mathrm{H}) ;{ }^{13} \mathrm{C}-\mathrm{NMR}\left(125 \mathrm{MHz}, \mathrm{CDCl}_{3}\right): \delta(\mathrm{ppm})=14.3(\mathrm{CH}), 18.5\left(\mathrm{CH}_{2}\right), 19.6(\mathrm{CH}), 24.8\left(\mathrm{CH}_{2}\right)$, $25.0\left(\mathrm{CH}_{2}\right), 25.6\left(\mathrm{CH}_{2}\right), 26.2\left(\mathrm{CH}_{2}\right), 26.3\left(\mathrm{CH}_{2}\right), 26.9\left(\mathrm{CH}_{2}\right), 27.3\left(\mathrm{CH}_{2}\right), 28.5(3 x \mathrm{CH}), 33.3\left(\mathrm{CH}_{2}\right), 34.1$ (CH), $34.3\left(\mathrm{CH}_{2}\right), 36.6\left(\mathrm{CH}_{2}\right), 40.0(\mathrm{CH}), 53.6(\mathrm{CH}), 55.5\left(\mathrm{CH}_{3}\right), 55.7(\mathrm{CH}), 70.3(\mathrm{CH}), 77.3(\mathrm{CH}), 79.3$ 
(C), 155.9 (C); IR (ATR): v = 3447 (br m), 2930 (s), 2857 (m), 1686 (m), 1664 (m), 1399 (m), 1365

(m), 1173 (m), 1041 (s); MS (EI, $\left.180{ }^{\circ} \mathrm{C}\right): \mathrm{m} / \mathrm{z}(\%)=441\left(\mathrm{M}^{+},<1\right), 341$ (12), 298 (36), 280 (18), 236 (16), 212 (20), 196 (14), 168 (100); HR-MS ( $\left.\mathrm{C}_{25} \mathrm{H}_{47} \mathrm{NO}_{5}\right)$ : calcd. 441.3454, found 441.3463.

\section{S,3S,4aR,5R,8aS,5’S-3-(tert-Butyl-dimethyl-silanyloxy)-5-(5-methoxymethoxyoct-1-enyl)-2- methyloctahydroquinoline-1-carboxylic acid tert-butyl ester (25)}

$63 \mathrm{mg}(0.14 \mathrm{mmol}) 24$ was dissolved in $10 \mathrm{~mL}$ dichloromethane, treated with $89 \mathrm{mg}(0.21 \mathrm{mmol}) \mathrm{DMP}$ and stirred $2 \mathrm{~h}$ at rt. The remaining suspension was filtered over celite and evaporated in vacuo. The residue was dissolved in dichloromethane and filtered again over a short pad if silica and evaporated. The residue was re-dissolved in $2 \mathrm{~mL}$ methanol and cooled to $-55^{\circ} \mathrm{C}$. Then $11 \mathrm{mg}(0.28 \mathrm{mmol}) \mathrm{NaBH}_{4}$ was added, stirred for $3 \mathrm{~h}$ at $-55^{\circ} \mathrm{C}$ to $-40{ }^{\circ} \mathrm{C}$, filtered and evaporated in vacuo. Purification by chromatography in hexane/EtOAc = 8:2 gave $63 \mathrm{mg}(100 \%) 25$ as colourless oil .

$\mathbf{R F}($ hexane/EtOAc $=6: 4)=0.47 ;[\alpha]^{20}{ }_{\mathbf{D}}=+13.5^{\circ}\left(\mathrm{c}=0.91, \mathrm{CHCl}_{3}\right) ;{ }^{1} \mathbf{H}-\mathbf{N M R}\left(500 \mathrm{MHz}, \mathrm{CDCl}_{3}\right)$ : $\delta(\mathrm{ppm})=0.90-0.93(\mathrm{t}, J=7.2 \mathrm{~Hz}, 3 \mathrm{H}), 0.97-1.12(\mathrm{~m}, 2 \mathrm{H}), 1.17-1.19(\mathrm{~d}, J=6.4 \mathrm{~Hz}, 3 \mathrm{H}), 1.21-1.43(\mathrm{~m}$, 16H), 1.45 (s, 9H), 1.64-1.66 (m, 1H), 1.76 (br s, 1H), 1.81-1.89 (m, 2H), 2.27-2.33 (m, 1H), 3.37 (s, 3H), 3.49-3.54 (m, 1H), 3.62-3.67 (m, 1H), 3.95-4.00 (m, 1H), 4.18-4.23 (dd, $J=8.3,13.2 \mathrm{~Hz}, 1 \mathrm{H})$, $4.64(\mathrm{~s}, 2 \mathrm{H}) ;{ }^{13} \mathrm{C}-\mathrm{NMR}\left(125 \mathrm{MHz}, \mathrm{CDCl}_{3}\right): \delta(\mathrm{ppm})=14.3(\mathrm{CH}), 18.6\left(\mathrm{CH}_{2}\right), 24.4\left(\mathrm{CH}_{2}\right), 24.5\left(\mathrm{CH}_{2}\right)$, $25.5\left(\mathrm{CH}_{2}\right), 26.4\left(\mathrm{CH}_{2}\right), 26.9\left(\mathrm{CH}_{2}\right), 27.3\left(\mathrm{CH}_{2}\right), 28.5(3 x \mathrm{CH}), 30.1\left(\mathrm{CH}_{2}\right), 33.0\left(\mathrm{CH}_{2}\right), 33.4(\mathrm{CH}), 34.3$ ( $\left.\mathrm{CH}_{2}\right)$, $36.6\left(\mathrm{CH}_{2}\right), 39.9(\mathrm{CH}), 51.7(\mathrm{CH}), 54.8(\mathrm{CH}), 55.5\left(\mathrm{CH}_{3}\right), 66.8(\mathrm{CH}), 76.8(\mathrm{CH}), 77.2(\mathrm{CH}), 79.3$ (C), 155.9 (C); IR (ATR): v = 3452 (br, m), 2932 (s), 2857 (m), 1688 (s), 1665 (s), 1455 (m), 1398 (s), 1377 (s), 1365 (s), 1320 (m), 1040 (s); MS (EI, $\left.250{ }^{\circ} \mathrm{C}\right): \mathrm{m} / \mathrm{z}(\%)=411\left(\mathrm{M}^{+}-\mathrm{OCH}_{3},<1\right), 325$ (30), 307 (30), 299 (28), 281 (30), 263 (24), 168 (100); HR-MS $\left(\mathrm{C}_{24} \mathrm{H}_{45} \mathrm{NO}_{4}, \mathrm{M}^{+}-\mathrm{OCH}_{3}\right)$ : calcd. 411.3348, found 411.3377. 


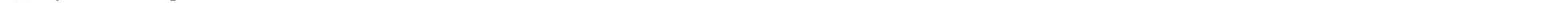




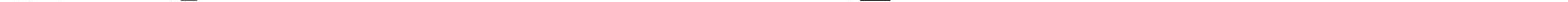




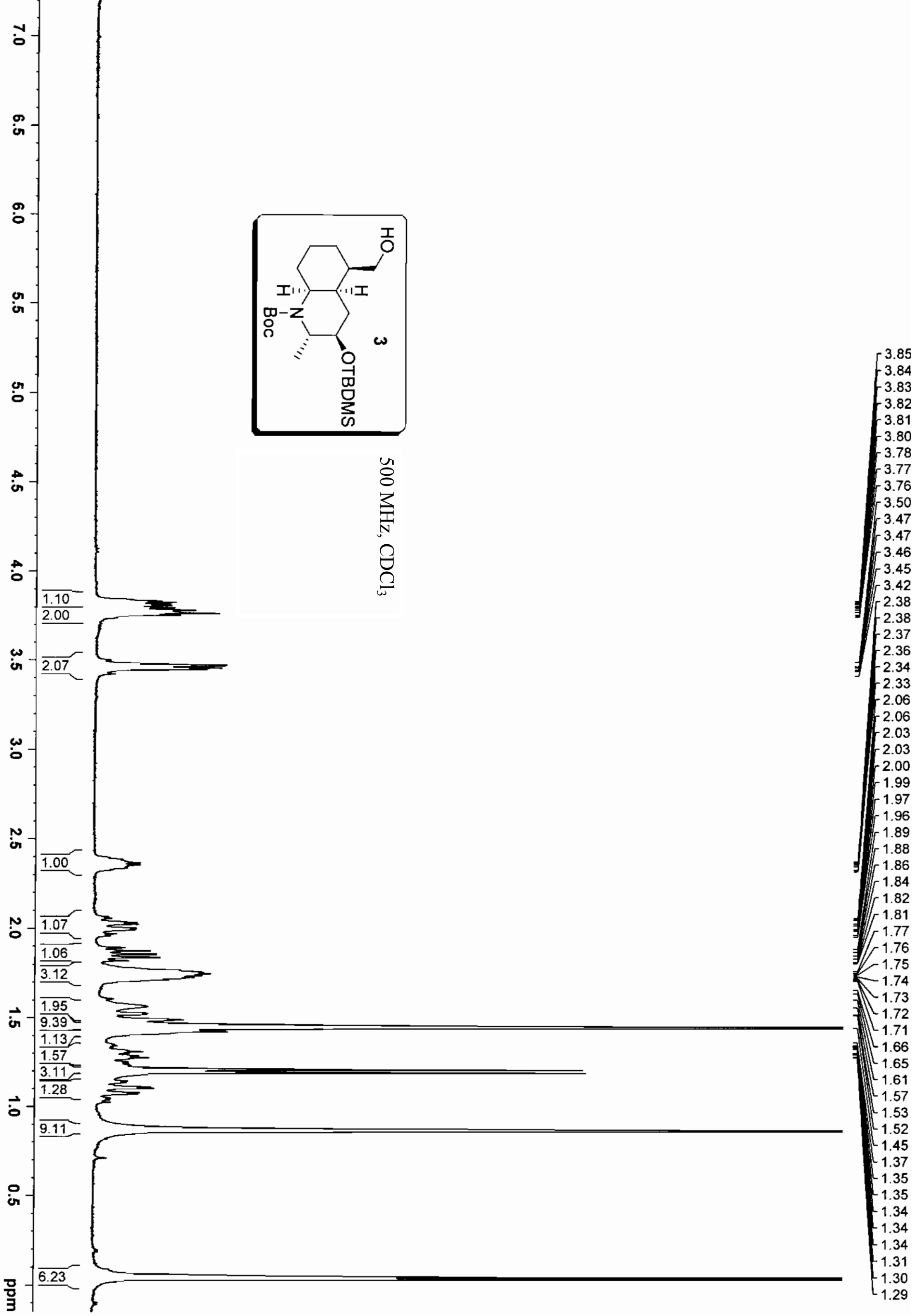




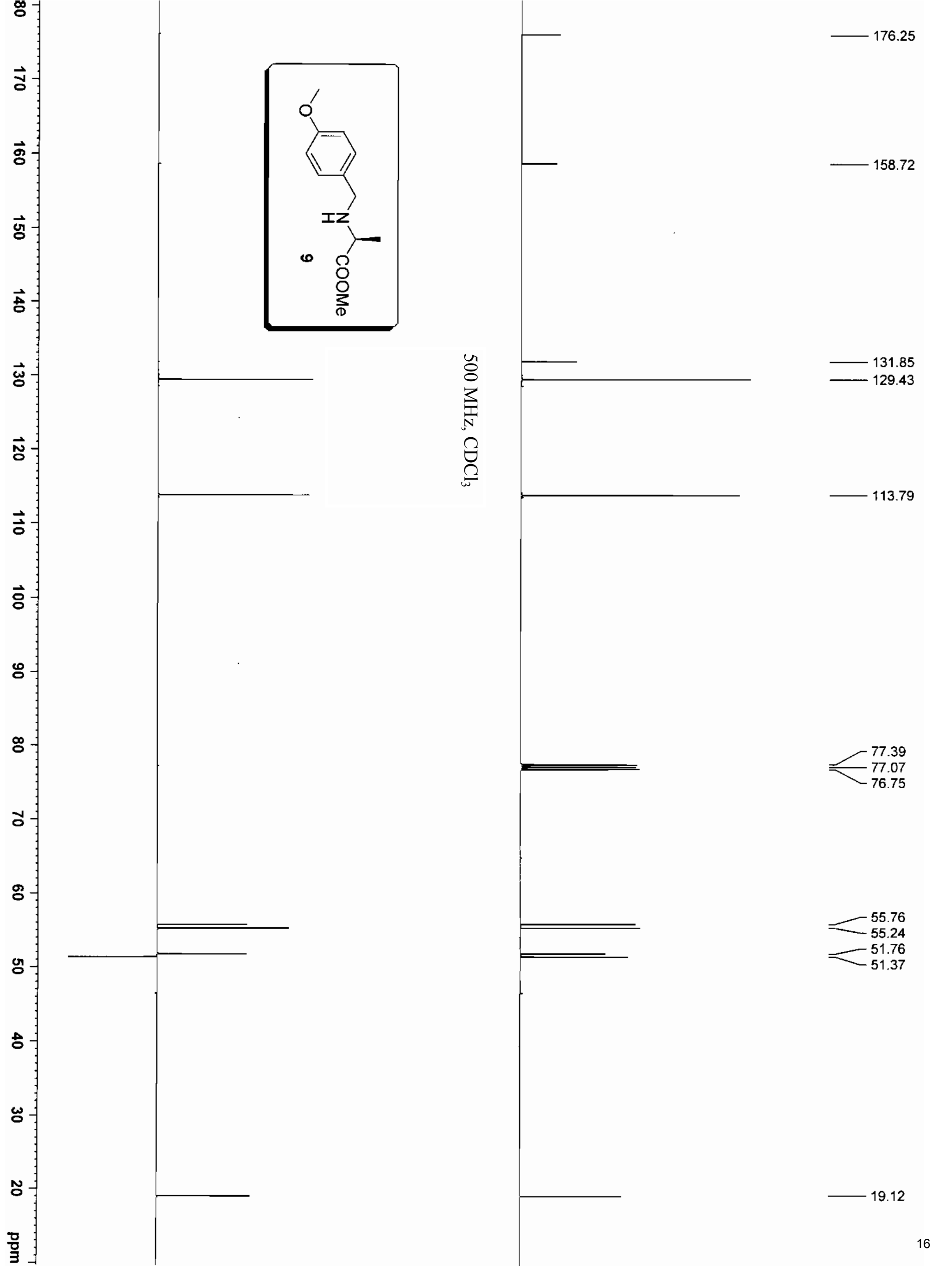




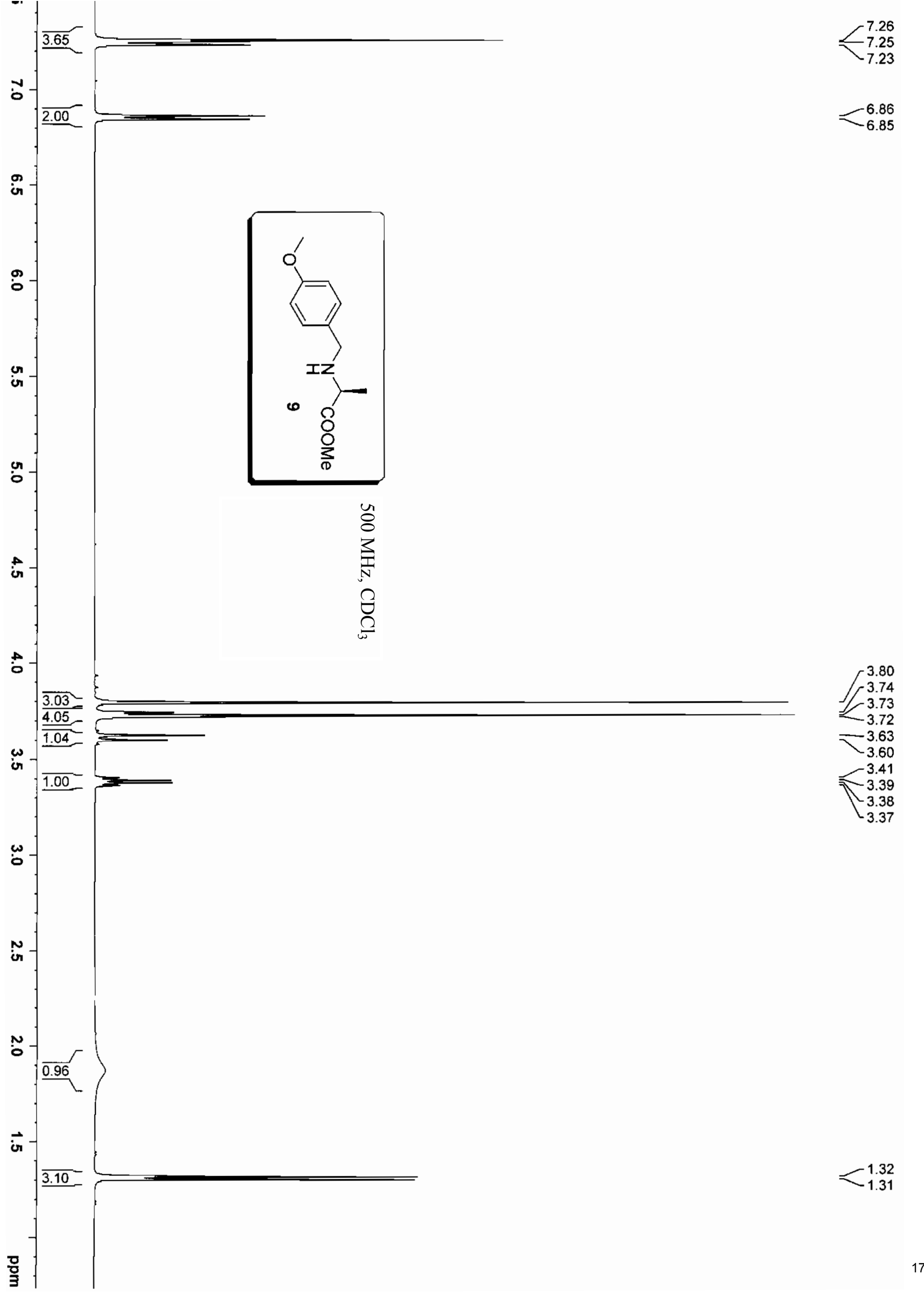




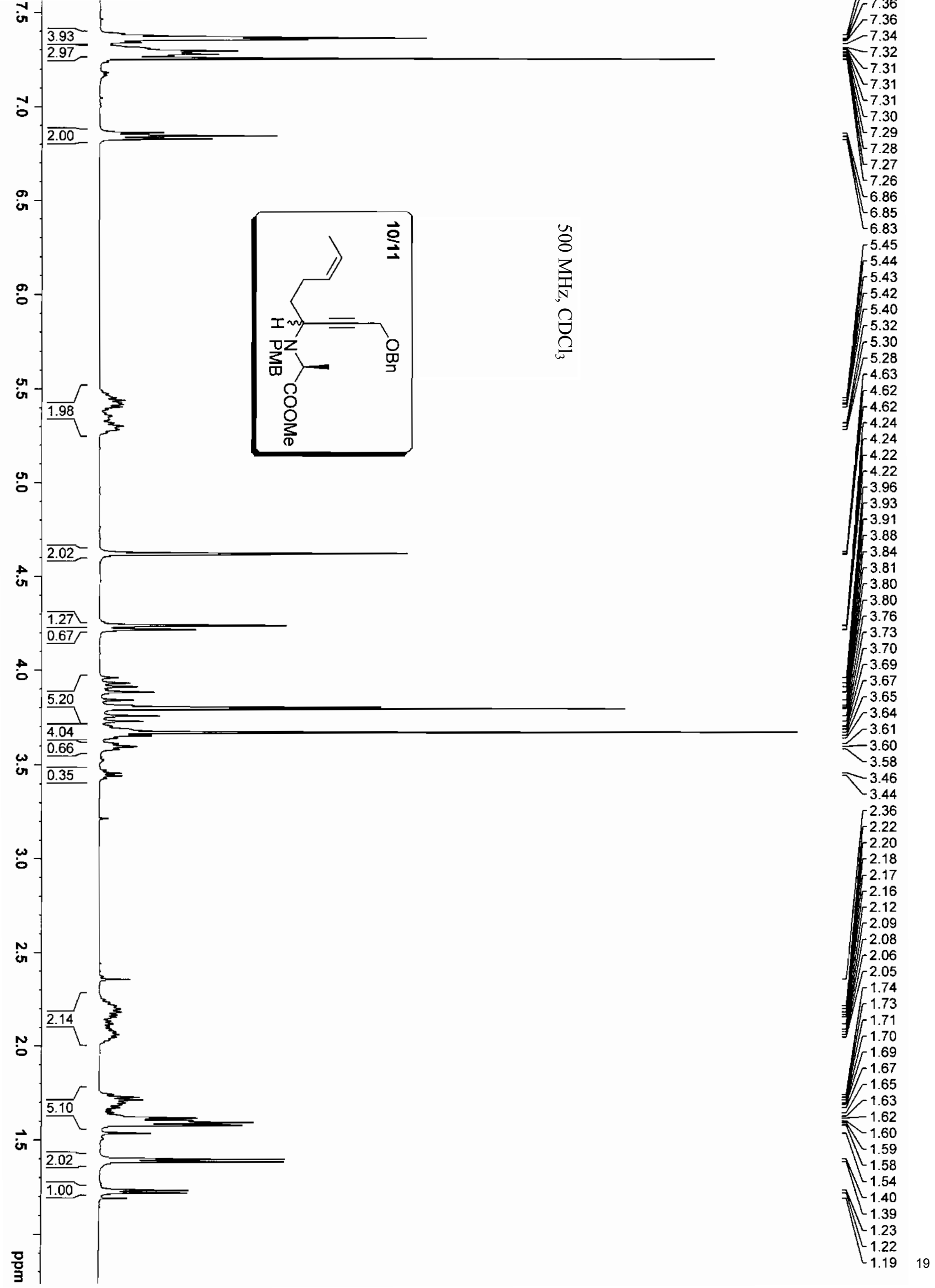




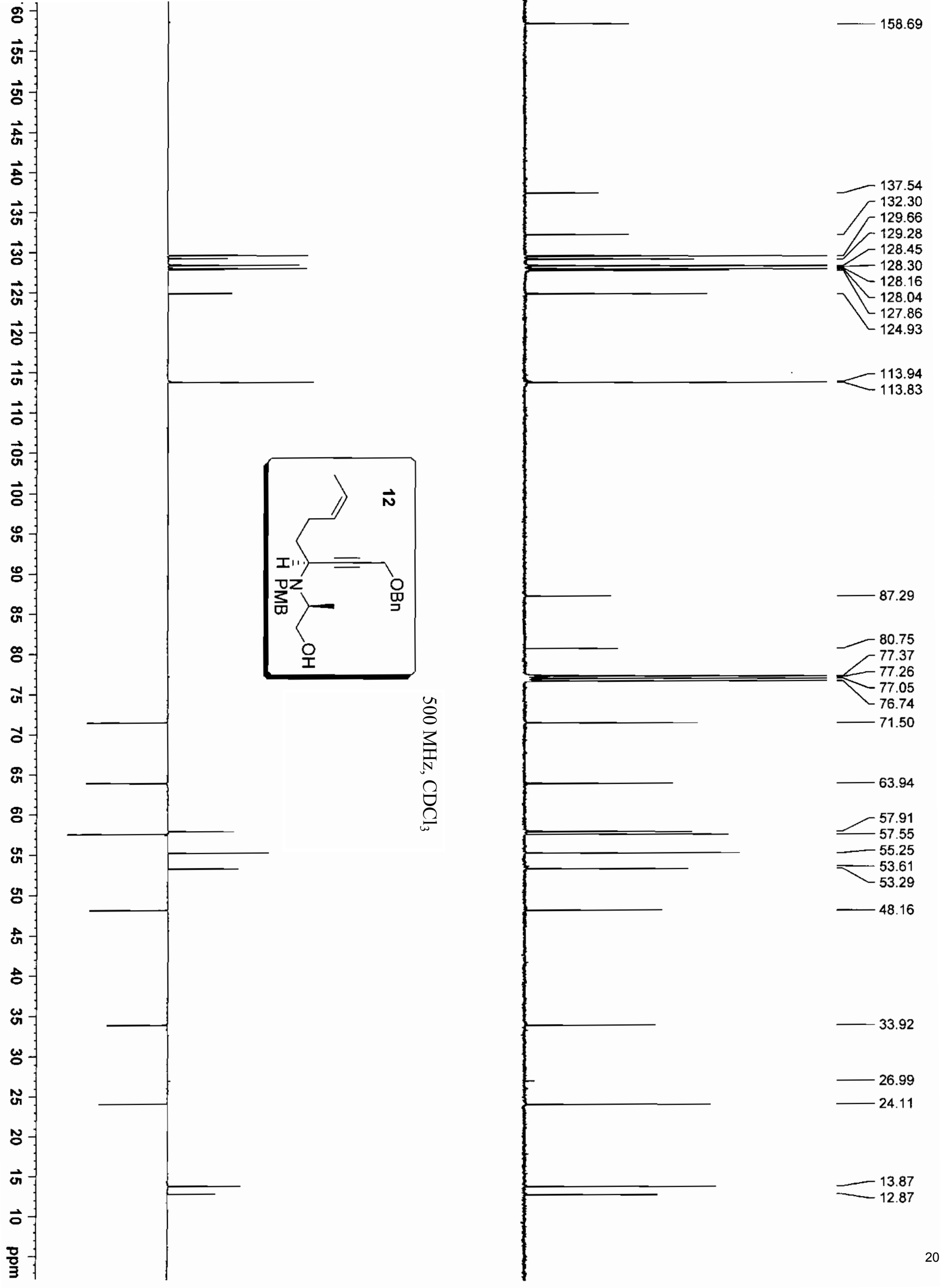




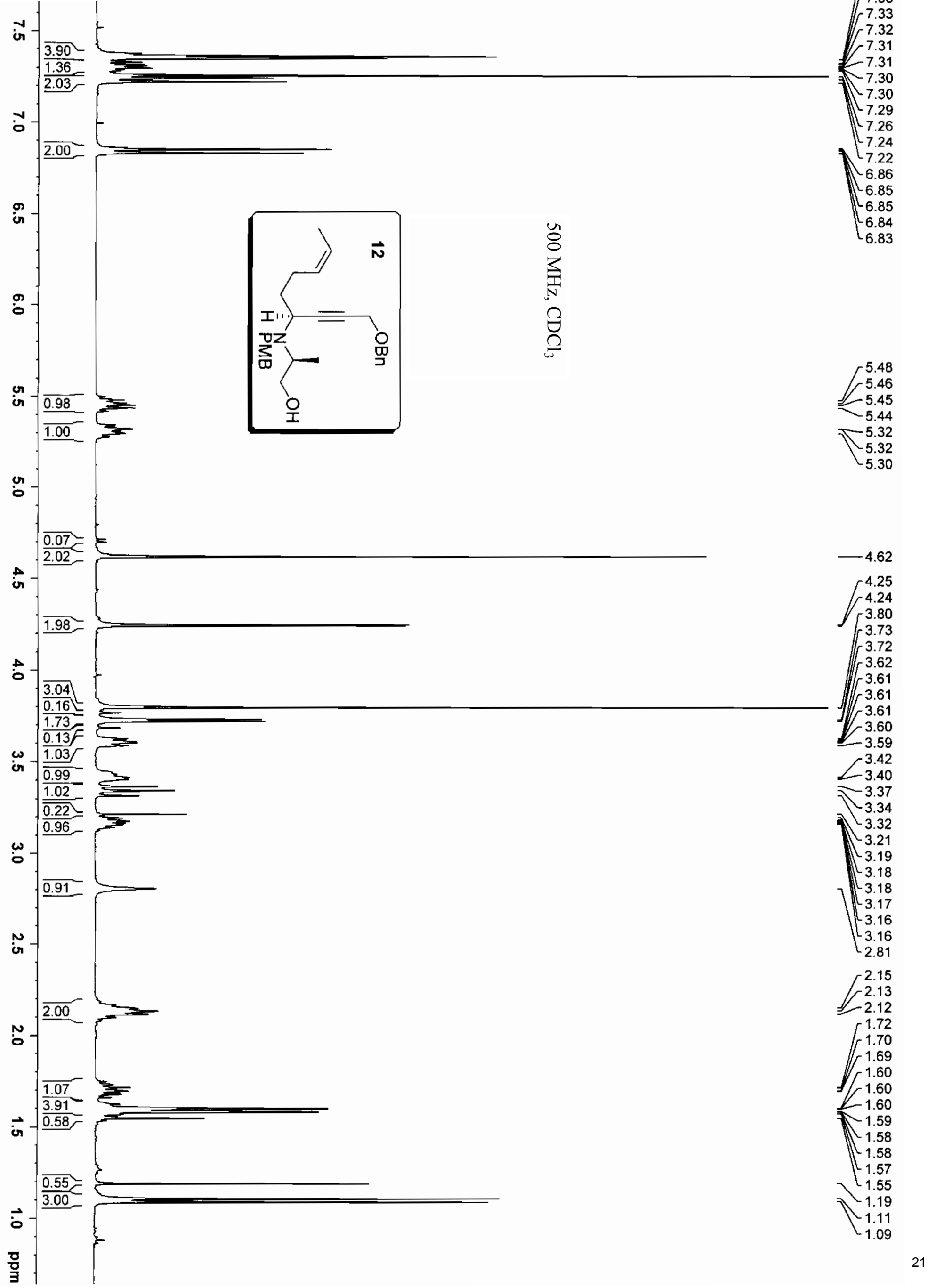




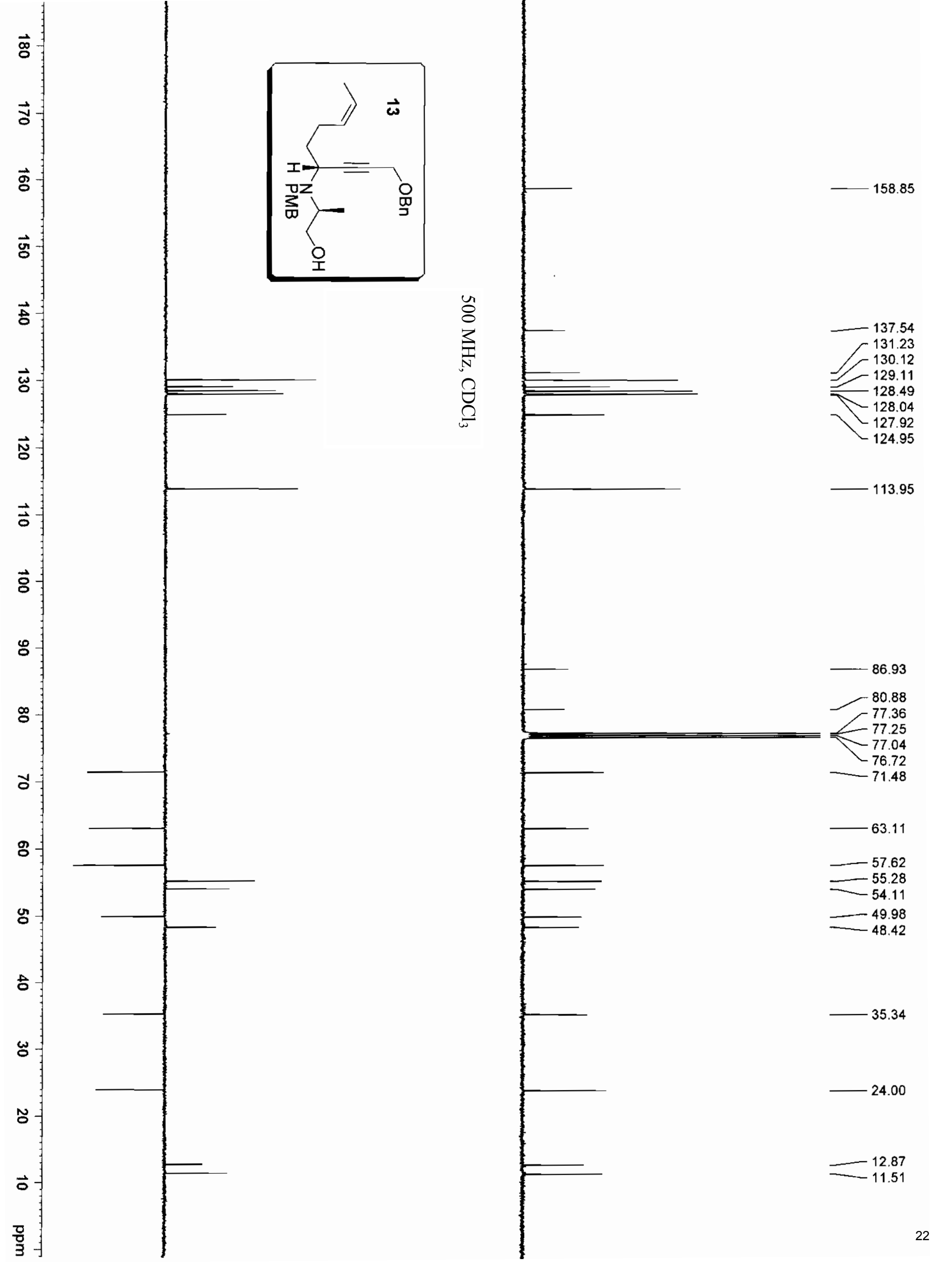




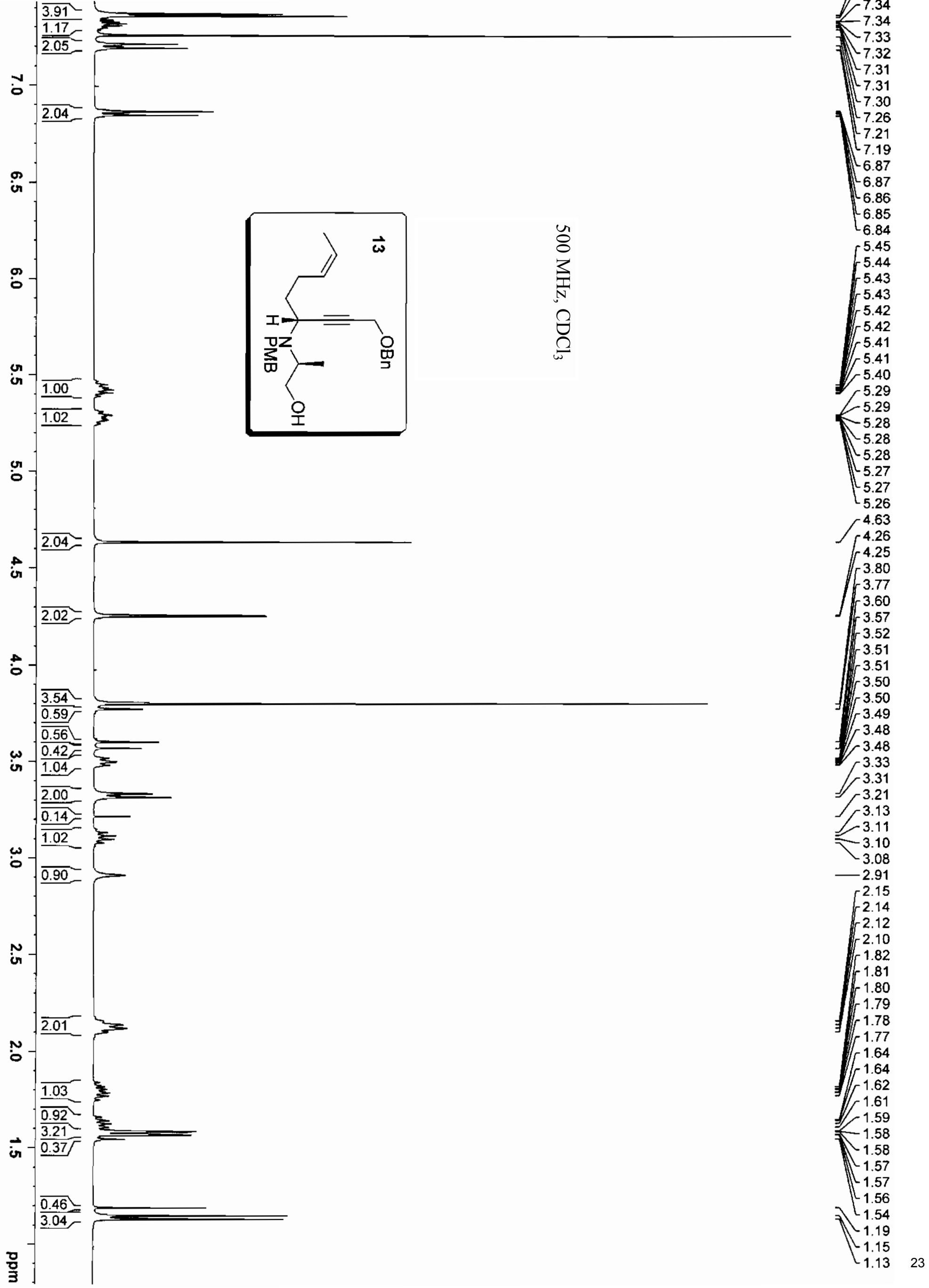




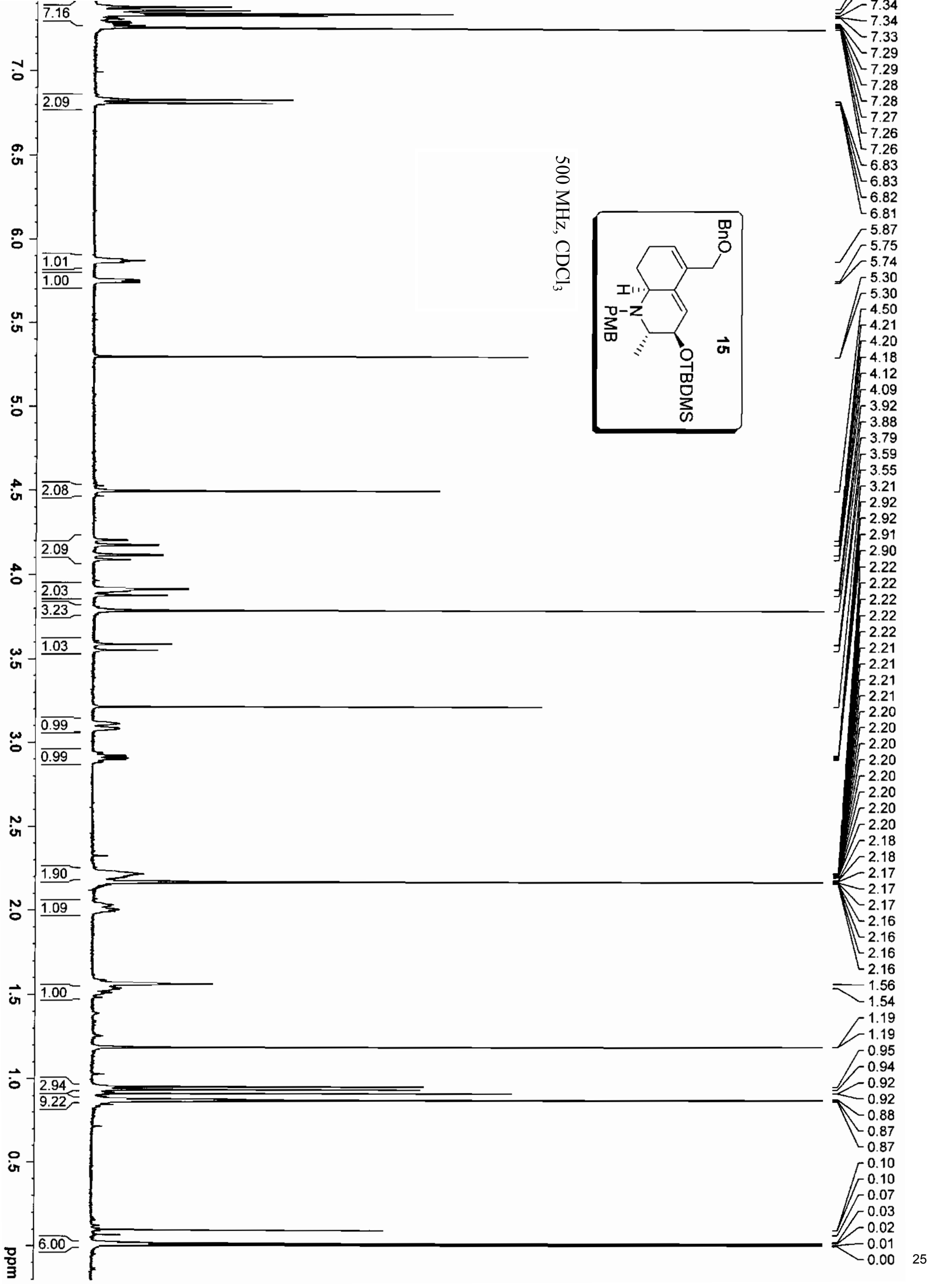




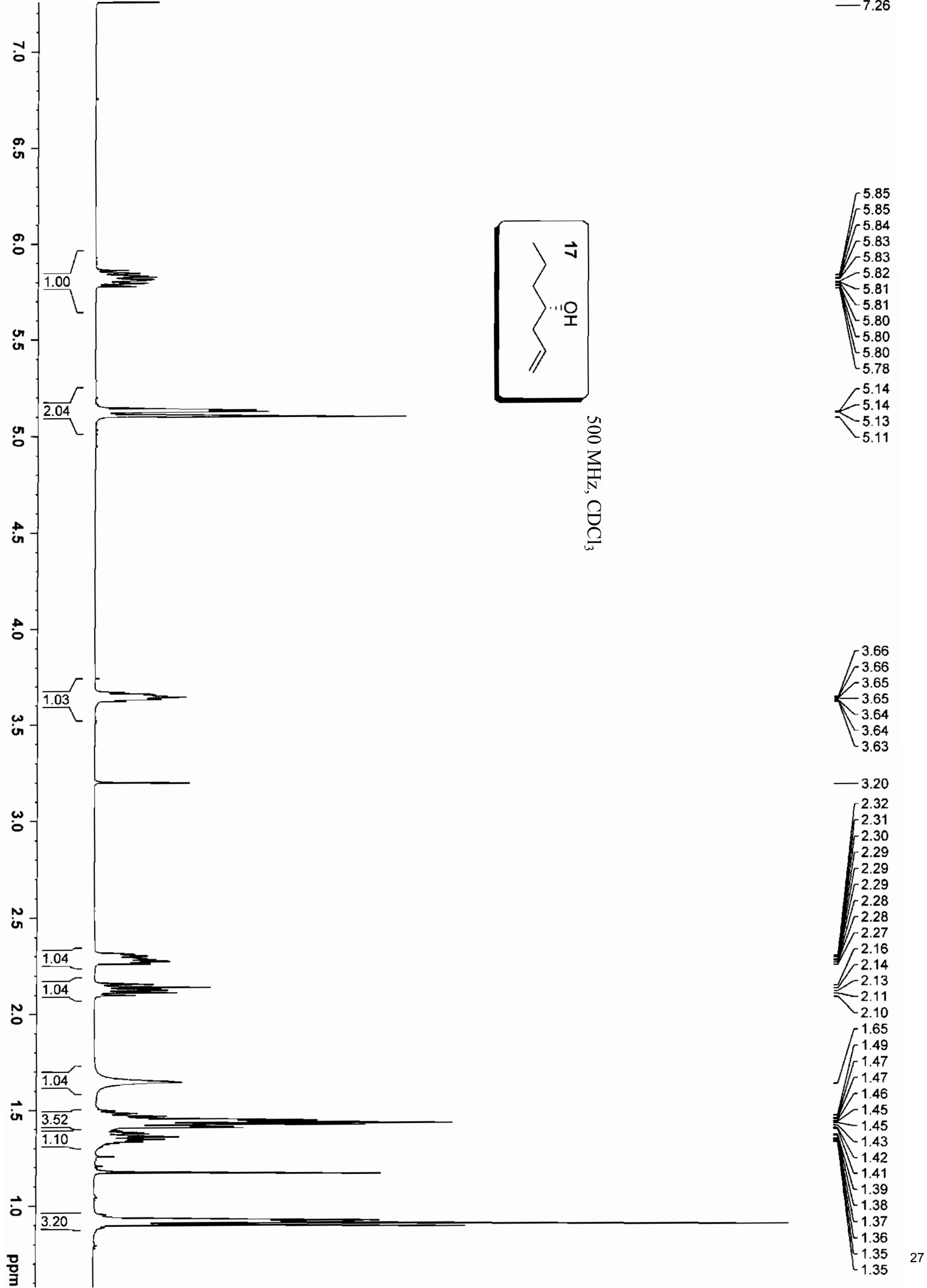




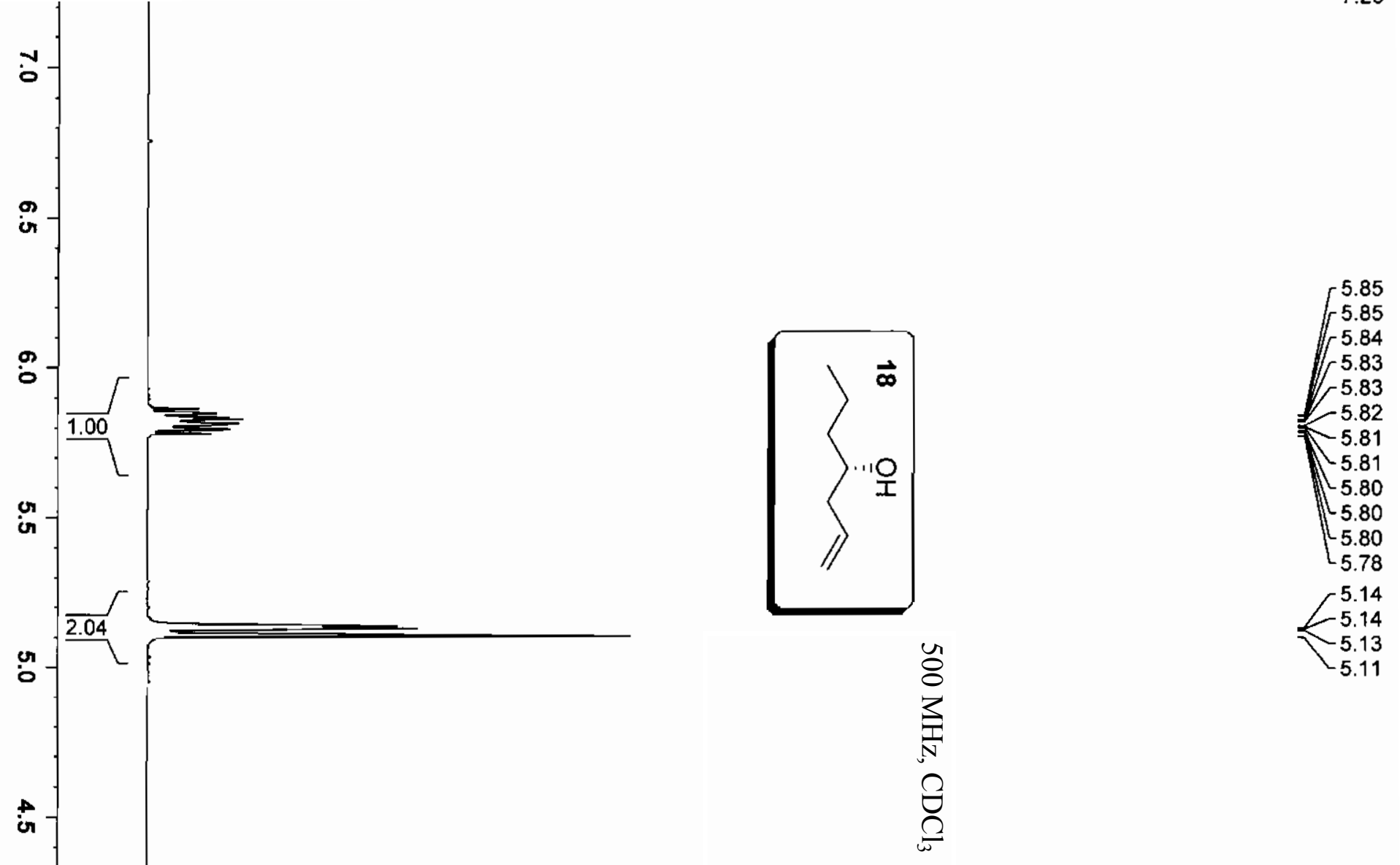




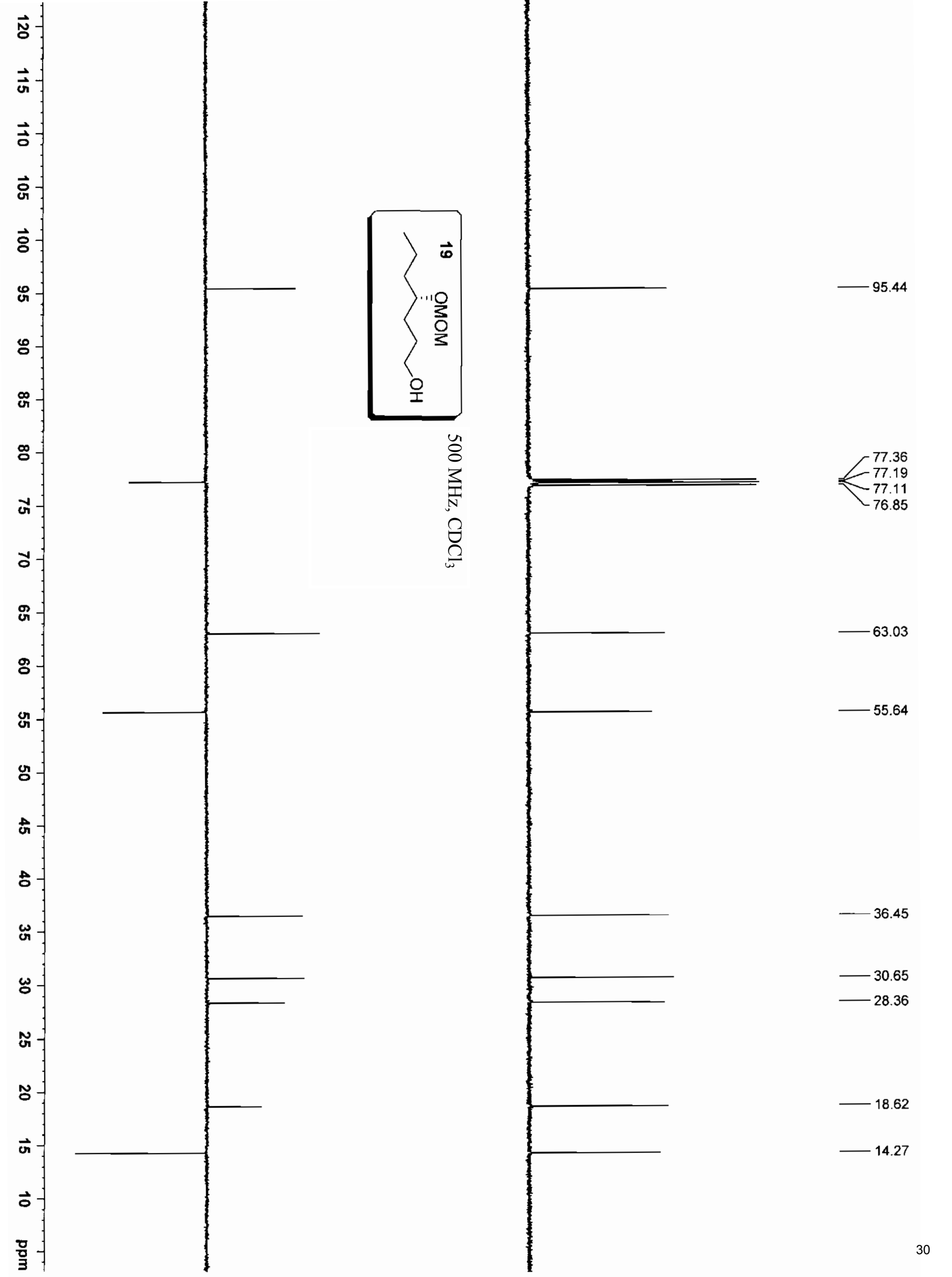




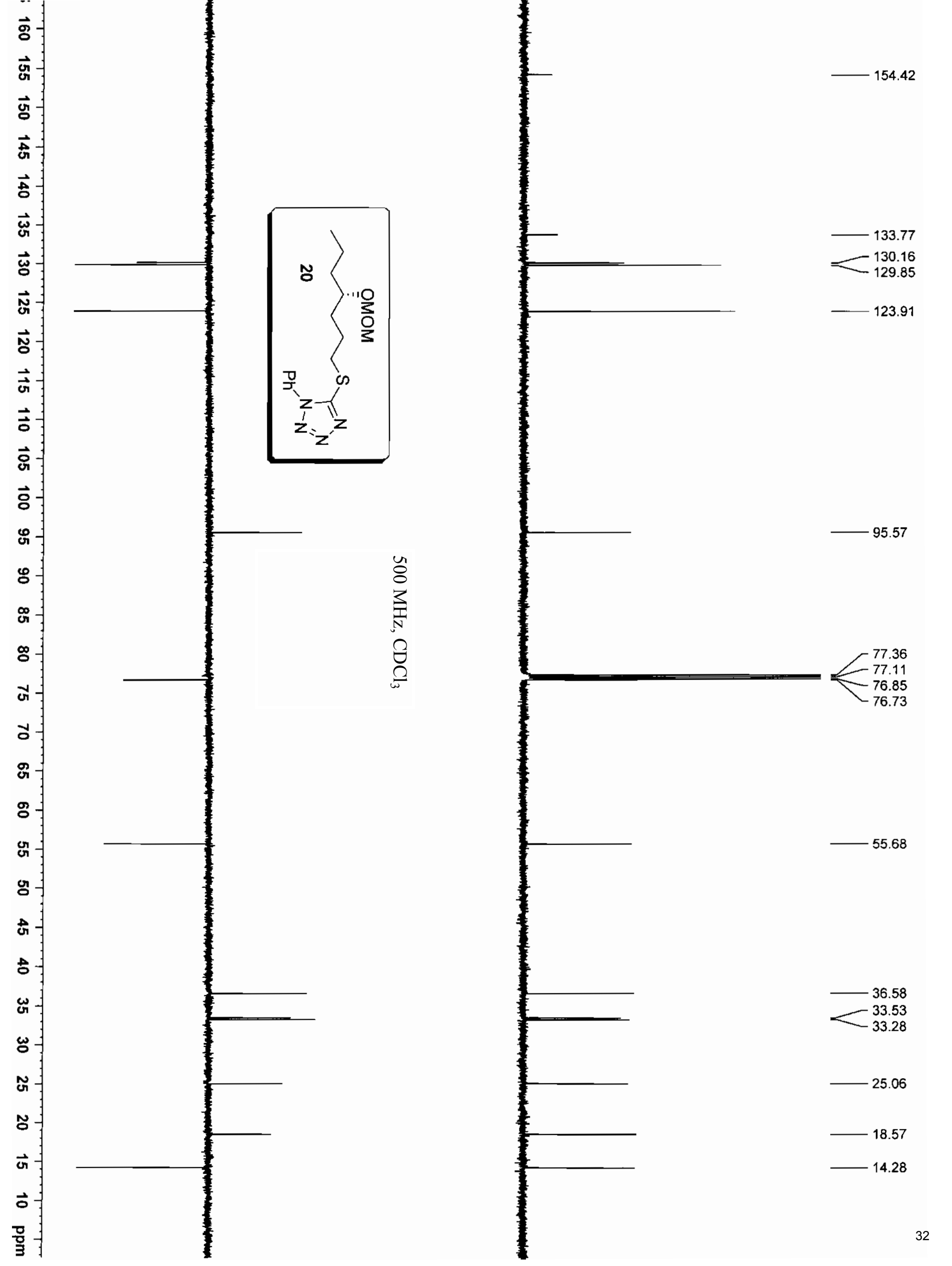




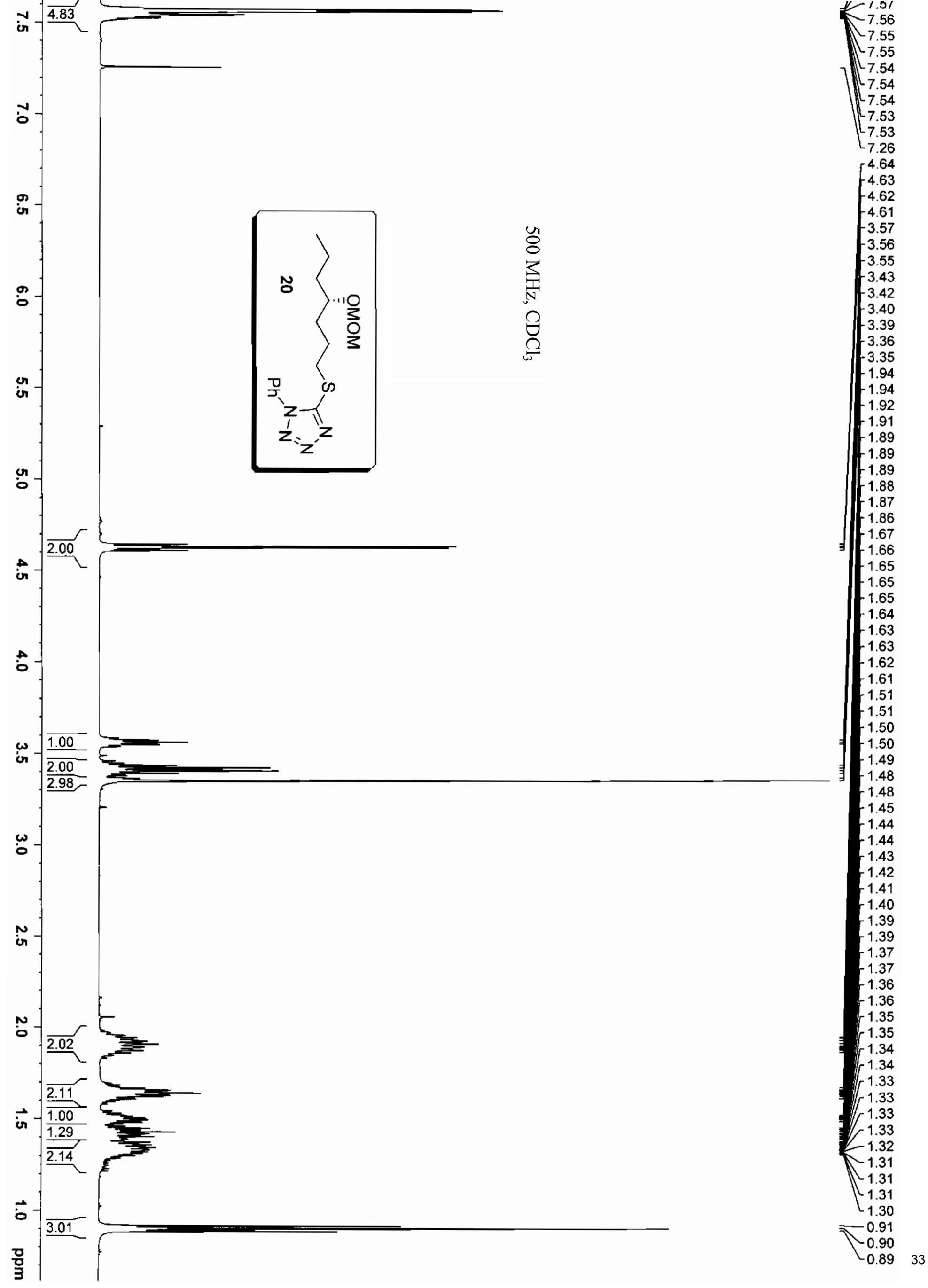




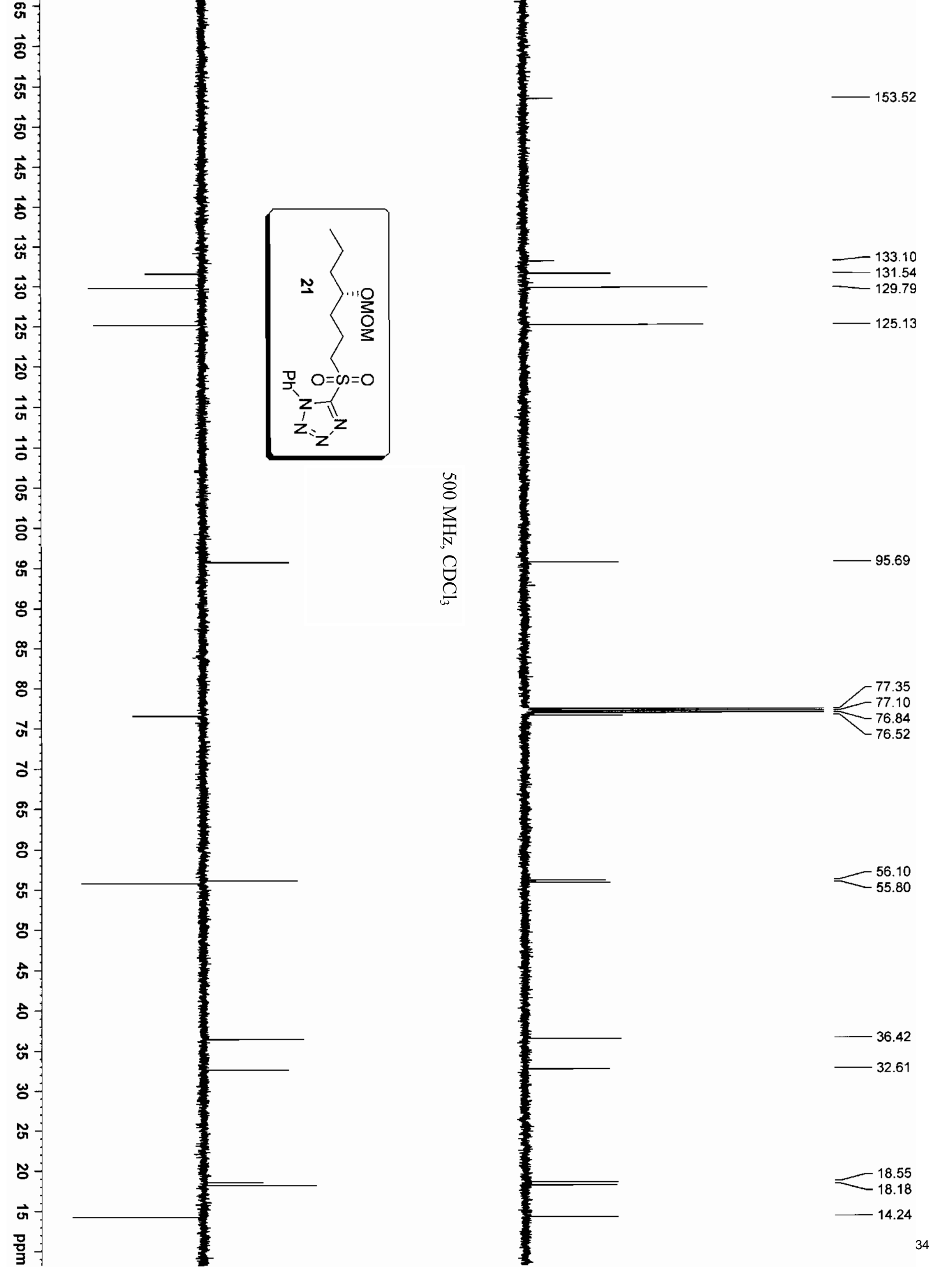




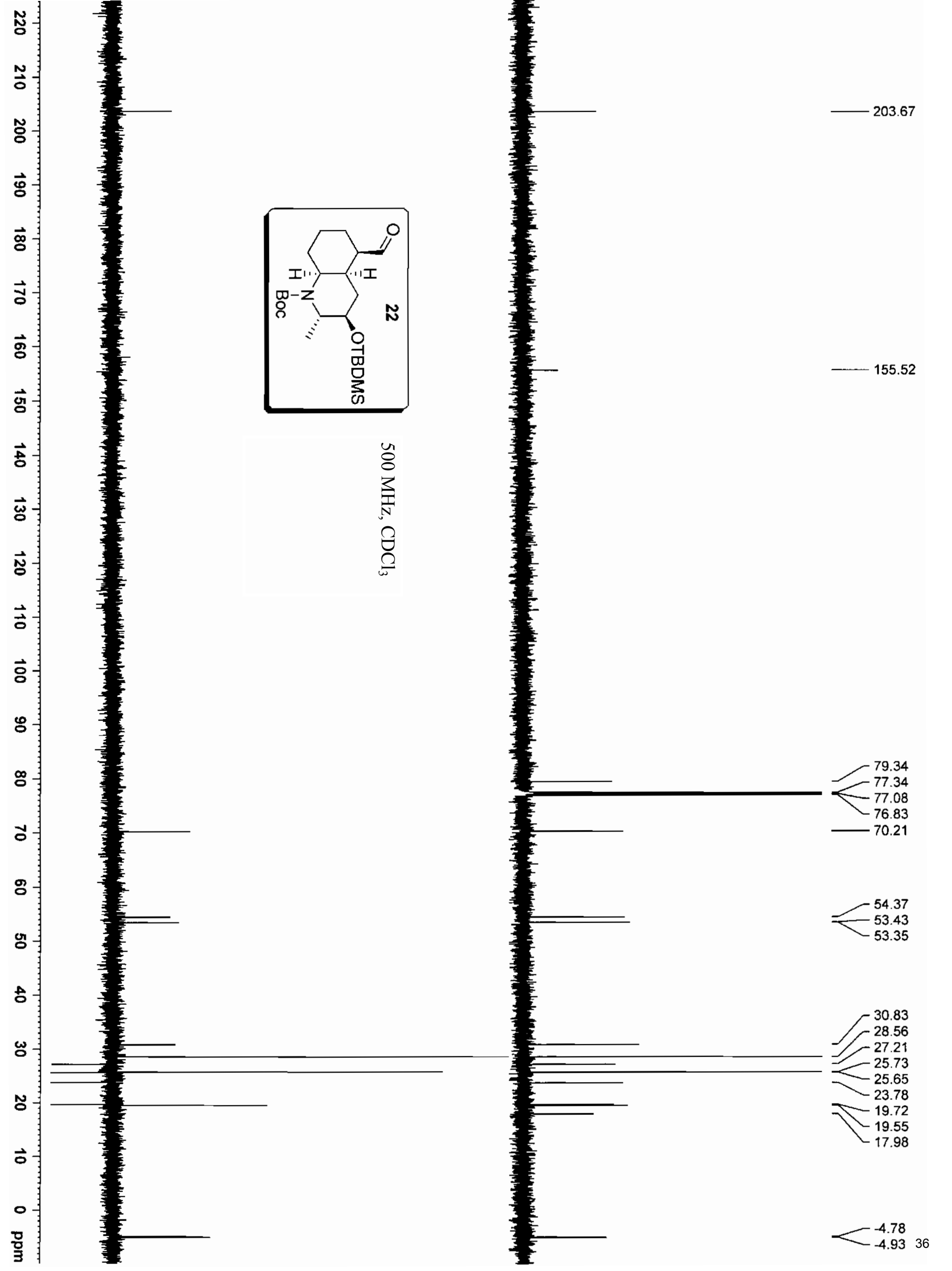




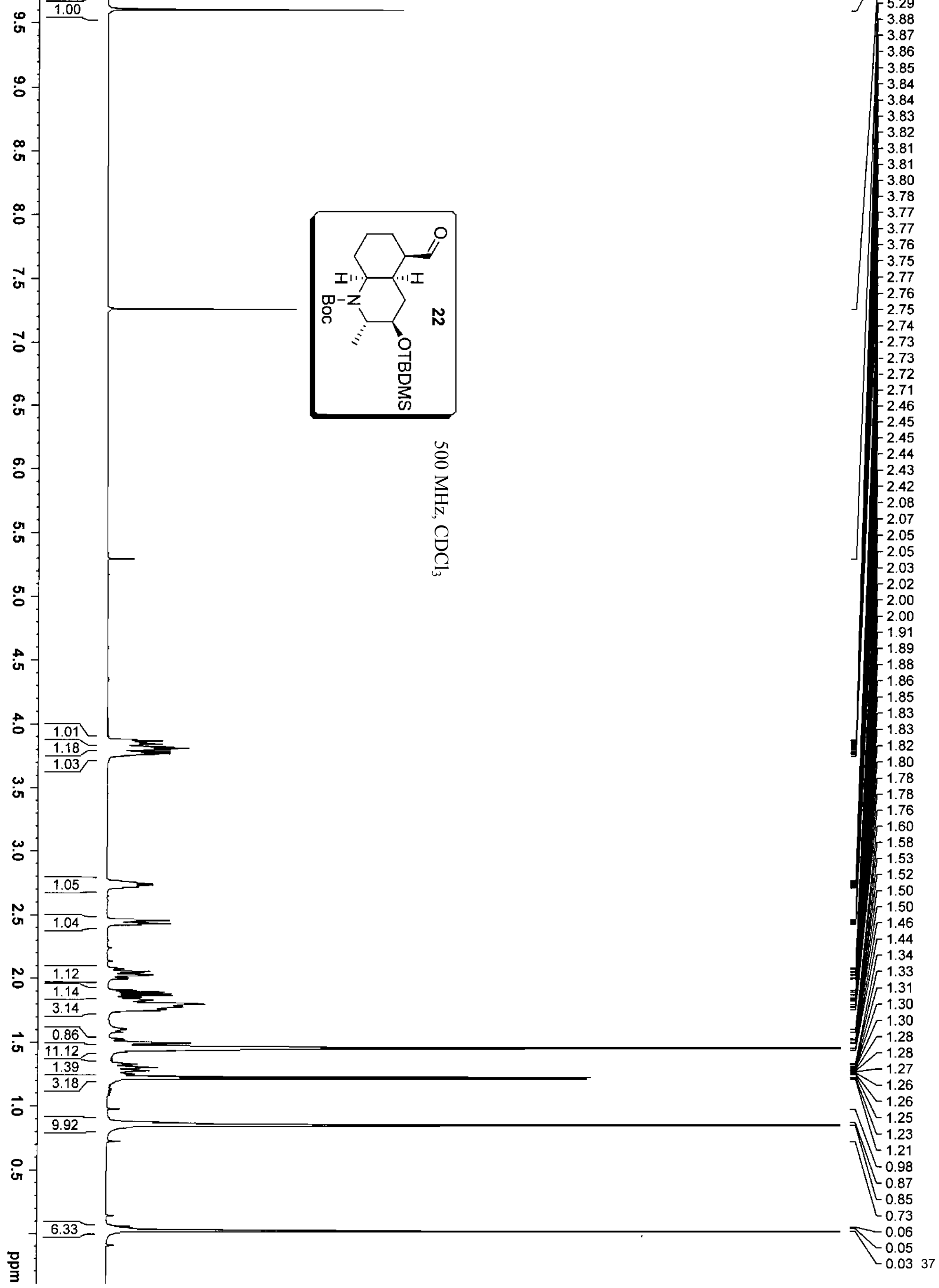




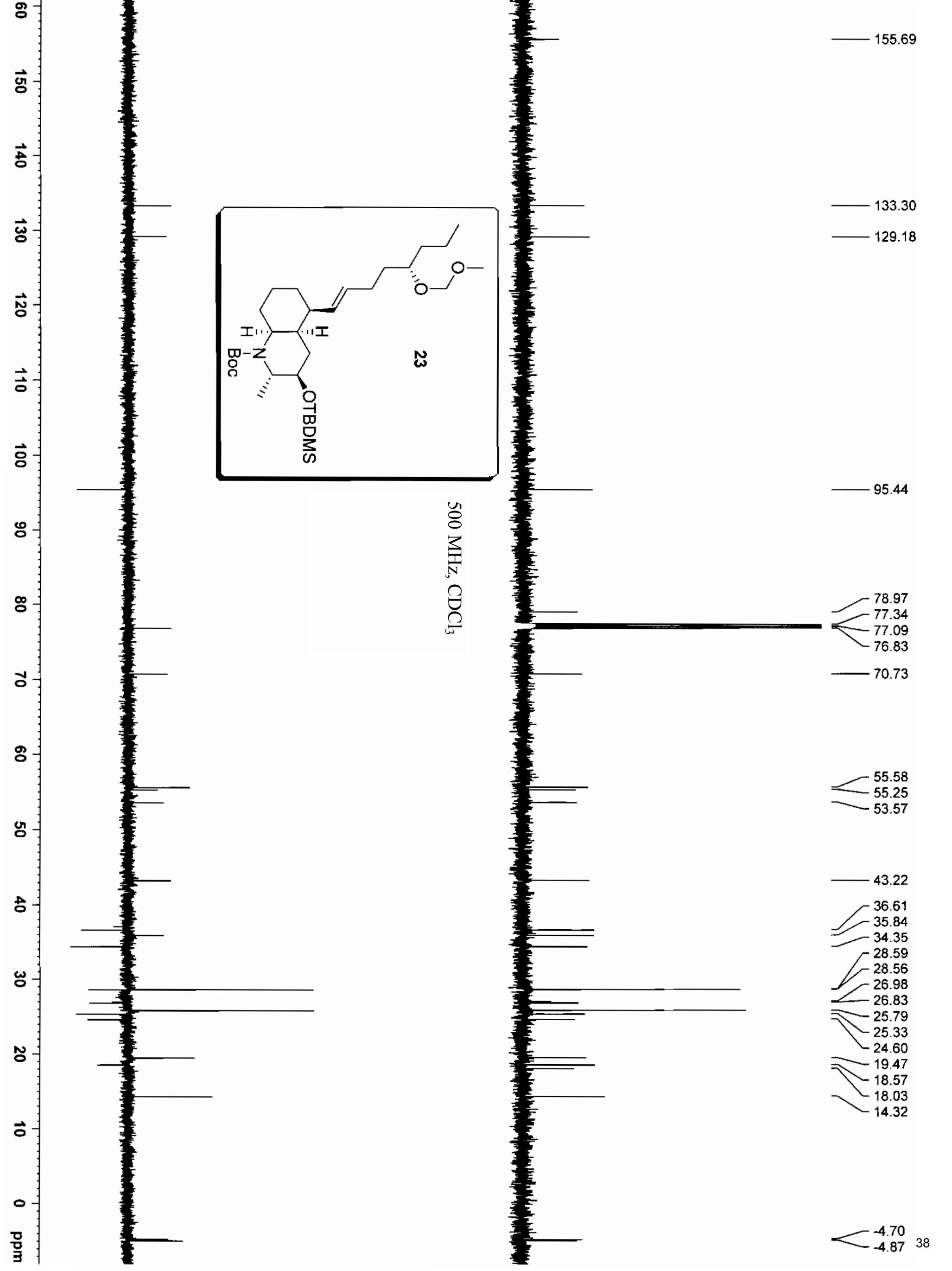




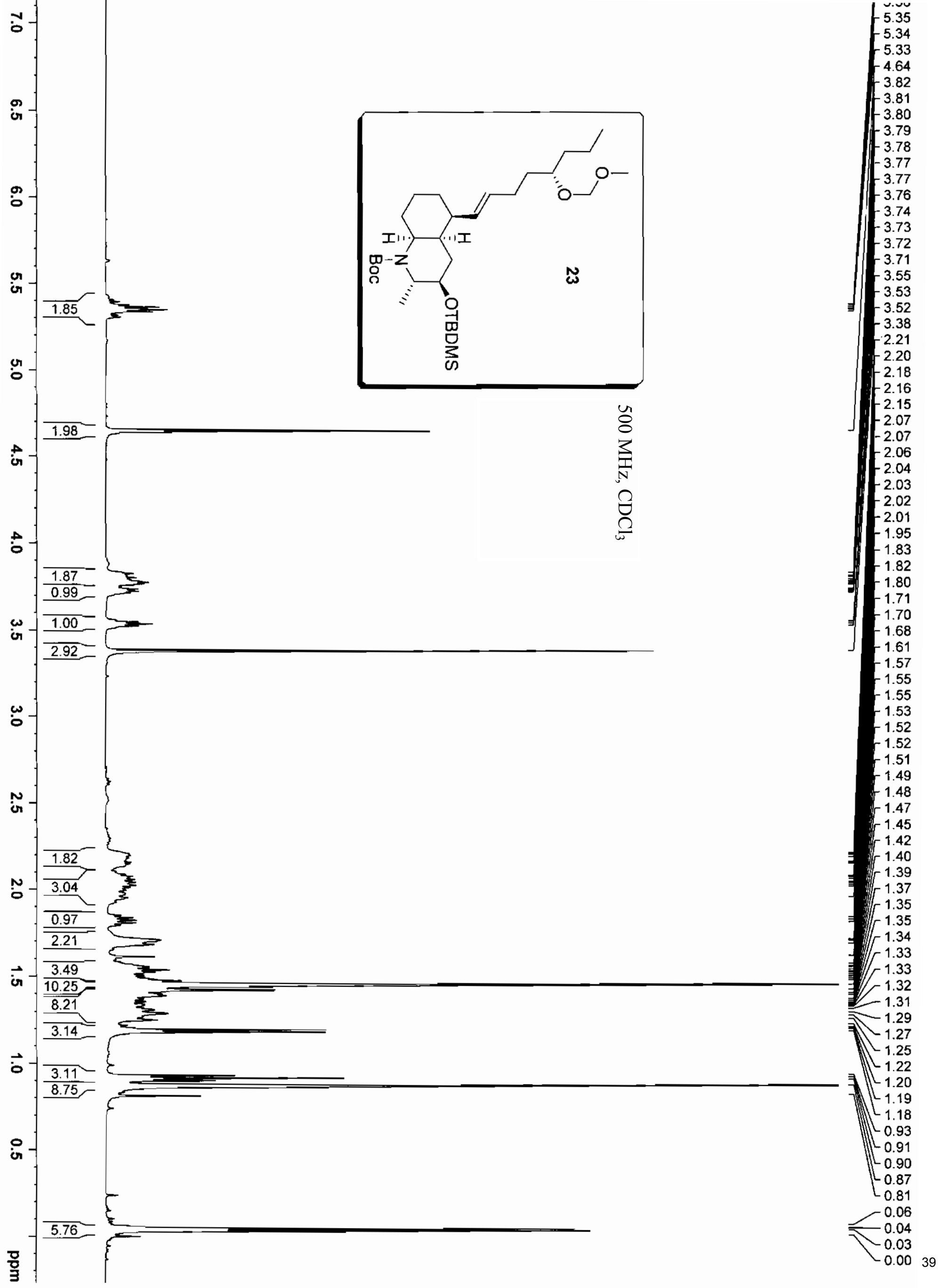




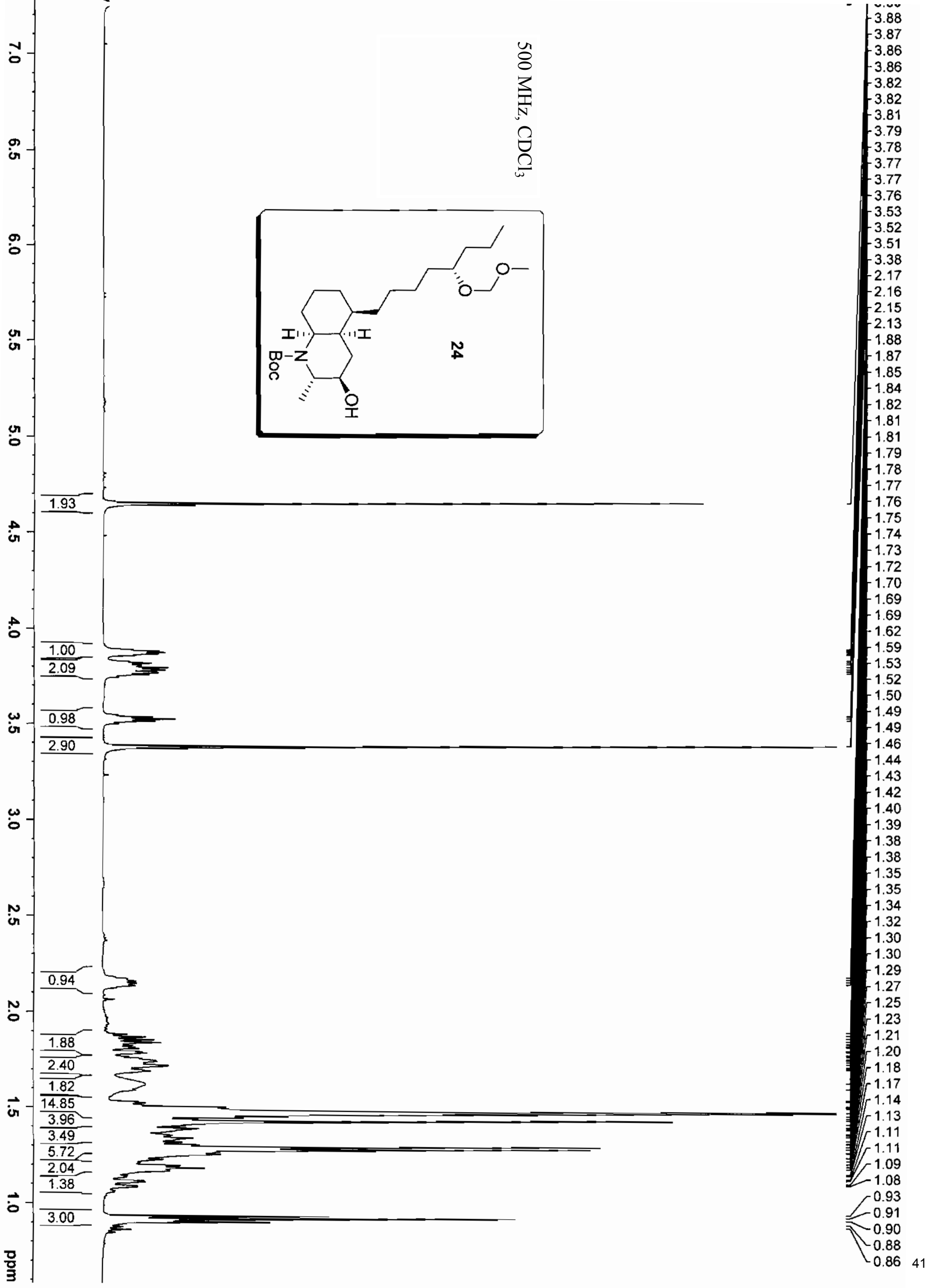




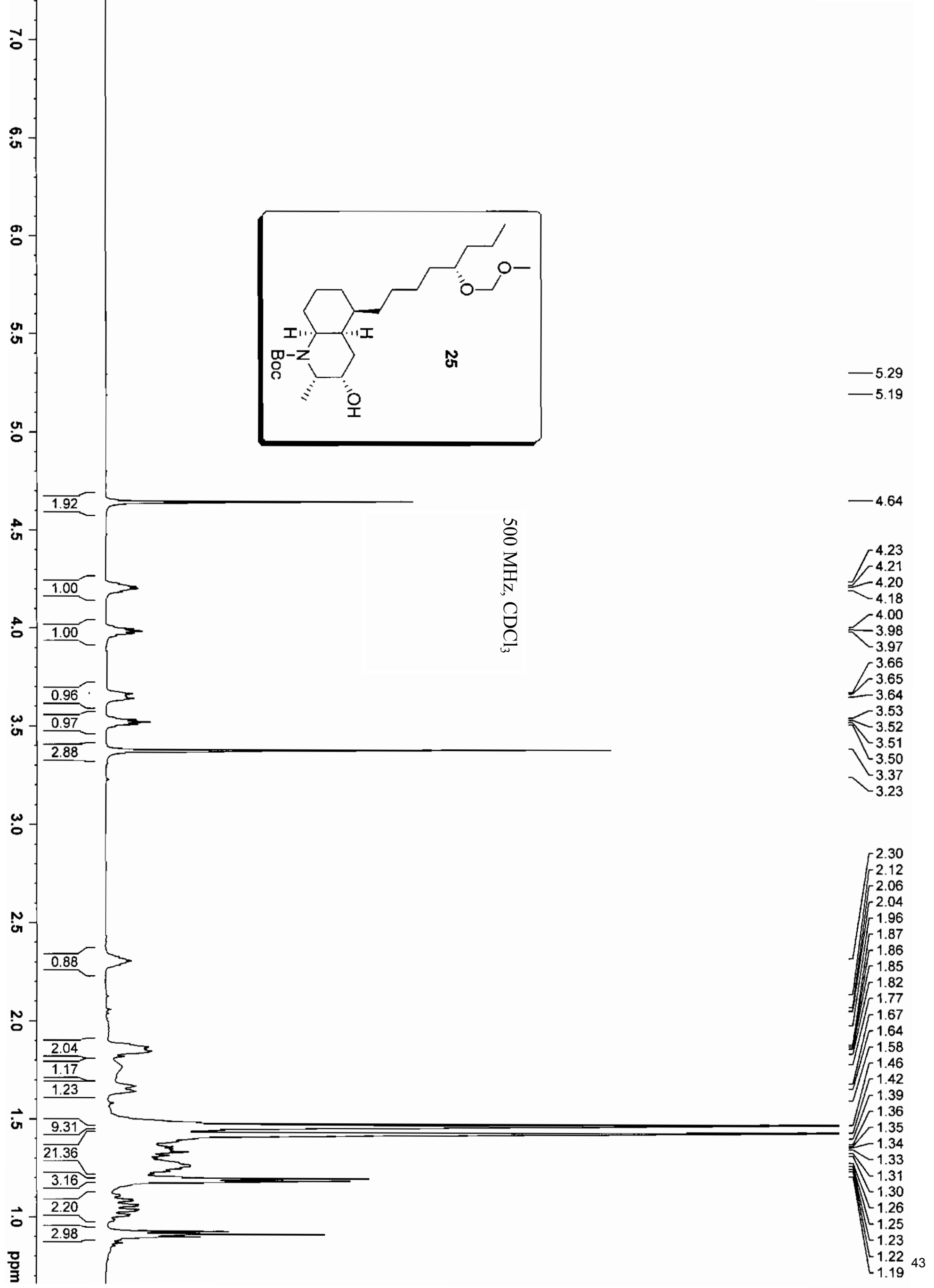

